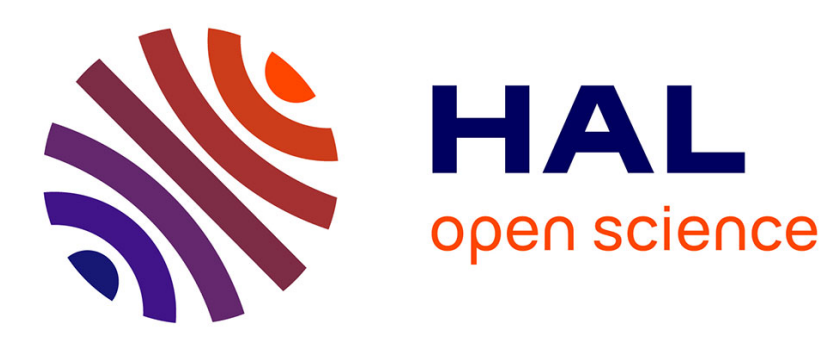

\title{
Comportements de départ en retraite et réforme de 2003: Les effets de la surcote
}

\author{
Samia Benallah
}

\section{To cite this version:}

Samia Benallah. Comportements de départ en retraite et réforme de 2003: Les effets de la surcote.

Economie et Statistique / Economics and Statistics, 2011, 10.3406/estat.2011.9614 . hal-02968989

\section{HAL Id: hal-02968989 \\ https://hal.science/hal-02968989}

Submitted on 16 Oct 2020

HAL is a multi-disciplinary open access archive for the deposit and dissemination of scientific research documents, whether they are published or not. The documents may come from teaching and research institutions in France or abroad, or from public or private research centers.
L'archive ouverte pluridisciplinaire HAL, est destinée au dépôt et à la diffusion de documents scientifiques de niveau recherche, publiés ou non, émanant des établissements d'enseignement et de recherche français ou étrangers, des laboratoires publics ou privés. 
Samia Benallah

\section{Citer ce document / Cite this document :}

Benallah Samia. Comportements de départ en retraite et réforme de 2003. Les effets de la surcote. In: Economie et statistique, $n^{\circ} 441-442,2011$. Les systèmes de retraite et leurs réformes : évaluations et projections. pp. 79-99;

doi : https://doi.org/10.3406/estat.2011.9614

https://www.persee.fr/doc/estat_0336-1454_2011_num_441_1_9614

Fichier pdf généré le 28/06/2018 


\title{
Comportements de départ en retraite et réforme de 2003 Les effets de la surcote
}

\author{
Samia Benallah*
}

La réforme des retraites de 2003 a créé la surcote, un dispositif accordant une majoration de pension aux individus qui prolongent leur activité au-delà de la durée requise pour l'obtention d'une pension complète. Cette mesure a explicitement pour objectif d'inciter les futurs retraités à retarder leur départ en se maintenant en emploi. Elle ne peut toutefois produire cet effet que si les considérations monétaires occupent une place importante dans l'arbitrage travail-retraite. S'il semble évident que cet arbitrage intègre une composante financière, le poids de cette dernière reste en revanche un sujet de débat. Nous étudions les conséquences de l'introduction de la surcote sur les comportements de départ en retraite de la première génération concernée par ce dispositif, à partir de l'échantillon des assurés de la Caisse Nationale d'Assurance-Vieillesse et d'une méthode par appariement. La création de la surcote aurait eu un effet positif sur l'âge de départ en retraite et sur la probabilité de poursuivre une activité au-delà de 60 ans des assurés nés en 1944 concernés par le dispositif. Elle aurait ainsi provoqué un report de deux mois de l'âge de liquidation des droits à la retraite et une augmentation de $16 \%$ de la probabilité d'être en emploi après 60 ans. En élargissant cette étude à d'autres générations, l'impact positif de la surcote sur l'âge de la retraite est confirmé. 
$\mathbf{L}$ es différentes réformes des retraites qui se sont succédées en France depuis près de vingt ans ont tenté, d'une manière ou d'une autre, de relever l'âge moyen de départ en retraite. En 2003, le législateur a introduit un nouveau dispositif, la surcote, pour inciter financièrement les seniors à retarder leur sortie du marché du travail. Cette mesure résume bien la philosophie de la réforme de 2003. Cette dernière privilégie en effet la logique incitative, accorder des pensions de retraite plus élevées aux individus qui partent en retraite plus tardivement, et renvoie ainsi le recul du départ en retraite à la décision individuelle.

Cette mesure ne peut cependant produire l'effet escompté que si que les considérations monétaires occupent une place importante dans l'arbitrage travail-retraite. S'il semble évident que cet arbitrage intègre une composante financière, le poids de celle-ci reste en revanche un sujet de débat. Les conclusions des recherches empiriques sont en effet partagées. Les comparaisons internationales expliquent généralement en grande partie les comportements de départ en retraite au sein de chaque pays par les barèmes des systèmes de retraite (ou de préretraite) nationaux (par exemple Blöndal et Scarpetta, 1997 ; Gruber et Wise, 2004) alors que les analyses sur données en coupe transversale concluent pour la plupart à un impact limité du système de retraite sur les décisions de départ (pour une revue de la littérature sur ces analyses micro-économétriques, voir notamment Lumsdaine et Mitchell, 1999). Les conclusions des recherches profitant d'une variabilité introduite par une modification de la réglementation en matière de retraite sont également partagées. Certaines font état d'un impact nul du barème des retraites sur les comportements de départ, d'autres concluent au contraire à une forte inflexion des décisions de départ liées aux modifications des modalités de calcul des pensions. La question de l'impact des incitations financières à la prolongation d'activité sur les décisions de départ en retraite ne semble donc pas complètement tranchée.

L'objet de notre étude est d'apporter des éléments de réponse à cette question en étudiant les comportements de départ suite à la création en 2004 du dispositif de la surcote. Nous estimons ainsi la sensibilité des individus aux incitations financières à la prolongation d'activité en mesurant les effets d'un renforcement de celles-ci. Nous disposons pour cela d'un cadre particulièrement adapté. Il y a en effet une réglementation qui évolue et modifie en conséquence les modalités de calcul des pensions. Cela crée une variabilité interindividuelle totalement exogène qui permet d'isoler l'effet potentiel du barème des pensions sur les comportements de départ en retraite. Il est par conséquent possible de mettre en œuvre une évaluation ex post de ce dispositif en suivant l'évolution des décisions de retraite avec l'application de la surcote, par une méthode d'appariement. Cette méthode nécessite de disposer de données précises permettant notamment de distinguer les individus concernés par le dispositif de ceux qui ne le sont pas et de comparer les comportements de ces deux sous-groupes. Les données mobilisées dans le cadre de cet article répondent à de telles exigences. Il s'agit des informations issues des fichiers administratifs de la Caisse nationale d'assurance-vieillesse (Cnav).

\section{Modification des barèmes des systèmes de retraite et décisions de départ : une revue de la littérature empirique}

Les bouleversements démographiques en cours, défavorables à l'équilibre des régimes de retraite, ont conduit de nombreux pays à engager d'importantes réformes de leur système de retraite. Ces différents changements de réglementations ont modifié le barème des pensions, créant ainsi un cadre particulièrement propice à la mesure de l'élasticité des comportements de départ en retraite. Les évaluations ex post sur des « quasi-expériences naturelles » en matière de retraite se sont ainsi développées ces dernières années et aboutissent à des résultats très partagés (pour une présentation plus complète de ces évaluations, voir Benallah, 2010). Ces études, qui tentent d'établir un lien de cause à effet entre une modification des modalités de calcul des droits à pension et les comportements de départ en retraite observés, portent sur trois types de modifications différents.

Le premier type de modification est la baisse programmée du montant des pensions. Pour les États-Unis, Krueger et Pischke (1992) ont évalué l'impact d'une loi de 1977 qui entraîne une baisse importante et brutale du montant des pensions servies par la Social Security Administration (SSA). En théorie, une réduction du montant des droits à pension aurait dû conduire les assurés à repousser l'âge de leur départ en retraite (1). Or les auteurs montrent que, toutes choses égales par ailleurs, l'âge de

1. Repousser son départ en retraite permet en effet de maintenir ses revenus d'activité, à supposer que ces derniers soient supérieurs aux droits à pension. 
départ en retraite a continué de diminuer en dépit de la baisse du niveau de la pension de base organisée par la loi de 1977. Les auteurs en concluent que le barème des pensions n'a pas, en moyenne, d'influence significative sur les décisions de retraite.

L'assouplissement des conditions financières de cumul d'un emploi et d'une retraite constitue le second type de modifications réglementaires ayant donné lieu à des évaluations ex post. Dans un certain nombre de pays, et en particulier dans certains pays anglo-saxons, le cumul emploi-retraite a été rendu plus attractif financièrement à la suite d'une modification du plafond de revenus du travail qui donne droit à la perception d'une pension de retraite complète. Appelée « earnings test 》 (ou " earnings rule » en Grande-Bretagne), cette condition de revenus d'activité a ainsi été assouplie voire supprimée afin de favoriser le maintien en activité des travailleurs les plus âgés. Cette mesure a été particulièrement étudiée et a donné lieu à plusieurs évaluations ex post. Baker et Benjamin (1999) ont tout d'abord proposé une évaluation pour le Canada où la suppression de l'earnings test pour les personnes âgées de 65 à 69 ans a été organisée dès 1975 de manière séquentielle, selon la zone géographique. Au Royaume-Uni, l'earnings rule a été supprimé plus tardivement, en 1989, et l'évaluation du dispositif a été réalisée par Disney et Smith (2002). Plusieurs études ont également été menées aux États-Unis (Gruber et Orzsag, 2003 ; Song, 2003/2004 ; Song et Manchester, 2007a; Song et Manchester, 2007b; et Haider et Loughran, 2008) où la suppression de l'earnings test $\mathrm{s}$ 'est faite en deux temps : dans un premier temps elle a concerné les retraités âgés de plus de 70 ans (suppression en 1983) pour être ensuite élargie aux retraités âgés d'au moins 65 ans (en 2000). Sur la base des méthodes en doubles différences, les études menées aboutissent à des résultats plutôt mitigés. Si presque toutes les études concluent à une accélération de la liquidation des droits à la retraite suite à l'assouplissement des conditions de cumul emploi-retraite, aucune d'entre elles ne met en évidence un impact positif et significatif sur la probabilité d'être en emploi. En revanche, la suppression de l'earnings test semble avoir eu un effet positif sur le niveau des salaires perçus après l'âge de la retraite et une partie des études conclut également à un impact positif sur la durée du travail (augmentation du nombre d'heures travaillées ou de la probabilité de transiter d'une activité à temps partiel vers une activité à temps plein). Ces résultats laissent penser que l'assouplissement des conditions de cumul d'un emploi et d'une retraite n'a pas eu l'effet escompté sur l'emploi. En revanche, il semble avoir encouragé ceux qui étaient déjà en activité ou qui auraient de toute façon choisi de l'être après la liquidation de leur retraite, à accroître leur niveau de revenu en augmentant leur durée de travail.

Le troisième type de modifications réglementaires évalué est le renforcement du caractère incitatif au recul de l'âge de départ en retraite du barème des pensions. Deux types de renforcement ont été étudiés. D'un côté, Bozio (2006), Song et Manchester (2007b), Mastrobuoni (2009), Aubert (2009) et Baraton et alii (2010) profitent d'un durcissement des conditions d'ouverture d'une pension de retraite sans pénalité pour estimer l'élasticité de l'offre de travail aux incitations financières. Bozio, Aubert et Baraton et al. partent plus précisément du renforcement des incitations au recul de l'âge de départ issu des lois portant réforme des retraites en France de 1993 et de 2003. Ces dernières organisent en effet l'allongement progressif, par génération, de la durée de cotisation nécessaire à l'obtention d'une retraite sans pénalité. Bozio (2006) estime une élasticité de l'offre de travail de 1'ordre de 0,54 ce qui équivaut à un recul de l'âge de liquidation des droits à la retraite de 1,5 mois pour un trimestre requis supplémentaire. Les prolongements récents apportés à ce travail par l'auteur (Bozio, 2009) et l'évaluation proposée par Aubert (2009) renforcent cette conclusion. Ce dernier trouve également un impact positif de l'allongement de la durée d'assurance requise pour le taux plein sur la probabilité d'être en emploi dans les années qui précèdent l'âge minimal d'ouverture des droits à la retraite. Pour les fonctionnaires, l'étude de Baraton et al. (2010) confirme le rôle positif d'un allongement de la durée d'assurance requise sur l'âge de départ en retraite. Song et al. et Mastrobuoni étudient quant à eux l'impact du relèvement du Normal Retirement Age (NRA) qui est, pour la SSA aux États-Unis, l'âge à partir duquel la pension de retraite est servie sans pénalité. Une loi de 1983 a en effet imposé une augmentation progressive de deux mois par génération (à partir de la génération 1938) du NRA. Les deux études concluent à un impact significatif de l'augmentation du NRA sur l'âge de départ en retraite observé mais pas de même ampleur. Song et Manchester estiment que l'augmentation du NRA a entraîné une légère diminution de la probabilité de liquider sa pension de retraite avant le NRA (de l'ordre de deux à quatre points de pourcentage) alors que Mastrobuoni conclut à une augmentation d'un 
mois de l'âge effectif de départ en retraite pour chaque augmentation de deux mois du NRA, soit une élasticité de même ordre que celle mesurée par Bozio dans son étude de 2006. De l'autre côté, Pingle (2006) propose une mesure de l'élasticité de l'offre de travail aux incitations financières à partir de l'évaluation d'un dispositif de type majoration de pension pour les individus prolongeant leur activité au-delà d'un certain âge. Plus précisément, il évalue l'effet du renforcement progressif, aux ÉtatsUnis, du Delayed Retirement Credit (DRC) sur les comportements de départ en retraite en mesurant l'influence du niveau du DRC sur la probabilité d'être en emploi entre 65 et 69 ans. L'auteur aboutit à la conclusion que l'influence des incitations financières à la prolongation d'activité sur les comportements d'activité aux âges élevés est forte. Ainsi, une augmentation d'un point de pourcentage du niveau du DRC conduirait, d'après l'auteur, à une augmentation de même ampleur du taux d'emploi des hommes entre 65 et 70 ans.

L'importance du rôle du barème des pensions de retraite sur les décisions de départ est finalement très variable suivant le type de mesures étudié. Même si les études présentées ici portent sur des législations et des groupes très différents, il est possible de dégager plusieurs résultats importants. Si le montant des pensions ou encore les conditions de cumul d'un emploi et d'une retraite paraissent peu déterminants pour expliquer certaines évolutions des comportements de départ en retraite, les incitations financières à la prolongation d'activité semblent en revanche bien agir dans le sens attendu. L'ampleur des effets attribués à ces dernières reste toutefois très hétérogène. Dans la suite de cet article, nous proposons une nouvelle estimation de l'impact des incitations financières à la prolongation d'activité à travers la mesure de l'effet de la création de la surcote en France sur la première génération entièrement concernée par ce dispositif.

\section{La montée en charge du dispositif de la surcote}

Depuis son entrée en vigueur, la surcote (cf. encadré 1) concerne un nombre croissant de retraités (cf. graphique I) et les bénéficiaires de la mesure représentent $12,6 \%$ des nouveaux retraités de droit direct du régime général (2) en 2009. Au total, depuis sa création, près de 380000 assurés du régime général ont pu bénéficier de ce dispositif (bilan au 31 décembre 2010).

Cette augmentation de la part des « surcoteurs » s'accompagne d'un accroissement du nombre

2. Par opposition au droit dérivé, composé essentiellement de la pension de réversion.

\section{Graphique I}

Part des bénéficiaires de la surcote parmi les nouveaux retraités de droit direct entre 2005 et 2009

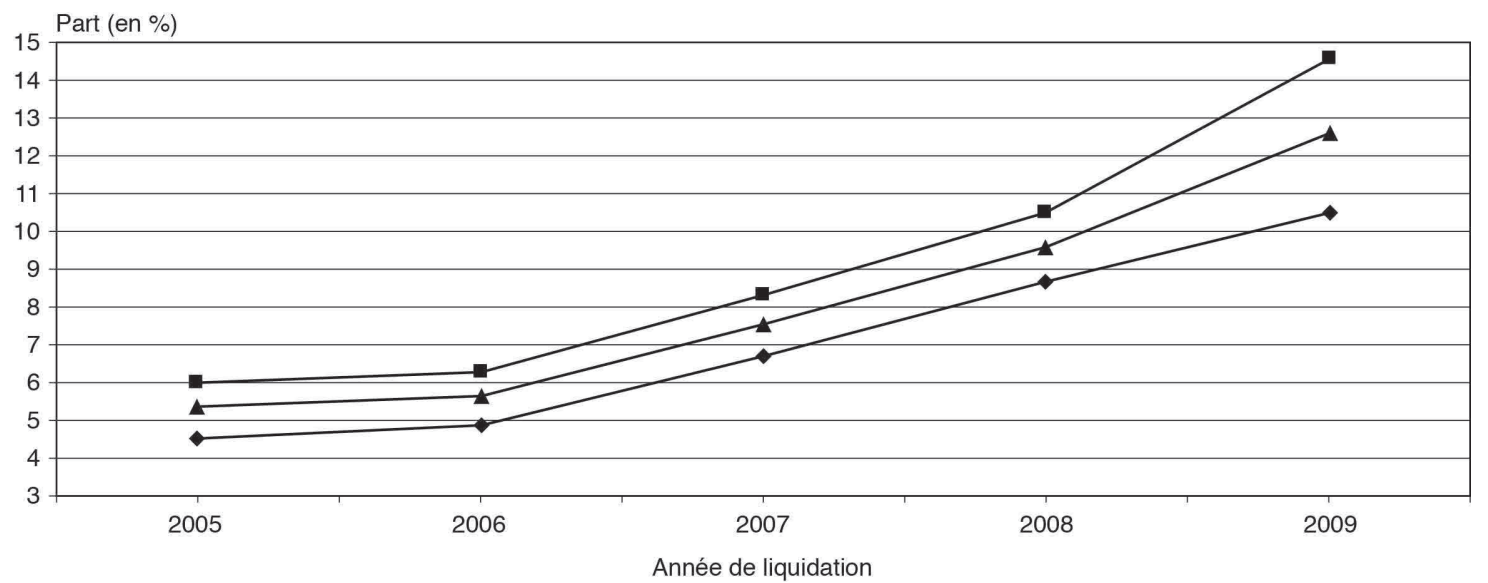

$\longrightarrow$ Hommes $\longrightarrow$ Femmes Ensemble

Lecture : 7,6 \% des assurés du régime général ayant liquidé leur pension en 2007 ont bénéficié de la surcote. Champ : ensemble des nouveaux retraités du régime général de 2005 à 2009.

Source : flux exhaustif des nouveaux retraités du Régime Général (RG), Système National Statistique des Prestations (SNSP, Infocentre). 
Encadré 1

\section{LE DISPOSITIF DE LA SURCOTE : PRÉSENTATION}

La prestation de retraite du régime général d'assurance-vieillesse est calculée sur la base d'une fraction de la moyenne des meilleures années de salaires perçus par l'assuré au cours de sa vie active. L'ampleur de cette fraction est variable. Elle dépend de la longueur de la carrière de l'assuré, la carrière étant ici entendue dans son sens large. On y intègre en effet toutes les périodes d'emploi mais également certaines périodes de chômage, de maladie et d'invalidité. Ces périodes sont converties en trimestres, et le nombre total de ces trimestres est appelé durée validée. Plus la durée validée par l'assuré est grande, plus ce dernier se voit attribuer une pension importante, jusqu'à un certain plafond, fixé à $50 \%$ de la moyenne des meilleures années de salaire. La réforme de 2003 a introduit un nouveau dispositif qui permet aux assurés qui souhaitent poursuivre leur activité au-delà de l'âge d'obtention des droits au taux plein d'améliorer leur pension en dépassant ce niveau plafond (article 25 de la loi n 2003/775 du 21 août 2003 créant l'article L. 3511-2 du code de la Sécurité sociale). Cette majoration de pension, couramment appelée surcote, s'ajoute, sous certaines conditions, à la pension de base pour les assurés qui cotisent plus de trimestres que nécessaire pour bénéficier d'une retraite sans pénalité. La surcote est appliquée directement au montant annuel brut de la pension, d'après le calcul suivant :

$P_{s}=$ Pension de base $\times \delta \times T R I M_{s}$
Le niveau de majoration $\delta$ dépend de la date d'effet de la pension et de la date de cotisation des trimestres supplémentaires (notés $T R I M_{s}$ ). Après sa mise en place, la surcote a en effet été renforcée par deux décrets (décrets $n^{\circ}$ 2006-1611 du 15 décembre 2006 et $n^{\circ}$ 2008-1509 du 30 décembre 2008 modifiant l'article D. 351-1-4 du code de la Sécurité sociale). La majoration de pension est ainsi déterminée selon les taux suivants :

- Si la pension de retraite du régime général prend effet entre le $1^{\text {er }}$ avril 2004 et le 31 décembre 2006, elle est majorée de $0,75 \%$ pour chaque trimestre supplémentaire cotisé après 60 ans et au-delà de la durée requise pour l'obtention du taux plein ;

- Si la date d'effet de la pension de retraite se situe entre le $1^{\text {er }}$ janvier 2007 et le 31 mars 2009, celle-ci est majorée de $0,75 \%$ du $1^{\text {er }}$ au $4^{\text {ème }}$ trimestre supplémentaire cotisé entre 60 et 64 ans et au-delà de la durée requise, de $1 \%$ à compter du $5^{\text {ème }}$ trimestre supplémentaire cotisé entre 60 et 64 ans et au-delà de la durée requise, et de 1,25\% pour chaque trimestre supplémentaire cotisé au-delà du $65^{\mathrm{ème}}$ anniversaire ;

- Si la date d'effet de la pension se situe après le $1^{\text {er }}$ avril 2009, la pension de retraite est majorée de 1,25\% pour chaque trimestre supplémentaire cotisé au-delà de la durée requise pour le taux plein à partir du $1^{\text {er jan- }}$ vier 2009 et après 60 ans. Pour les trimestres cotisés

\section{Graphique}

Taux théorique de pension en fonction de la distance au taux plein, avant et après la réforme des retraites de 2003

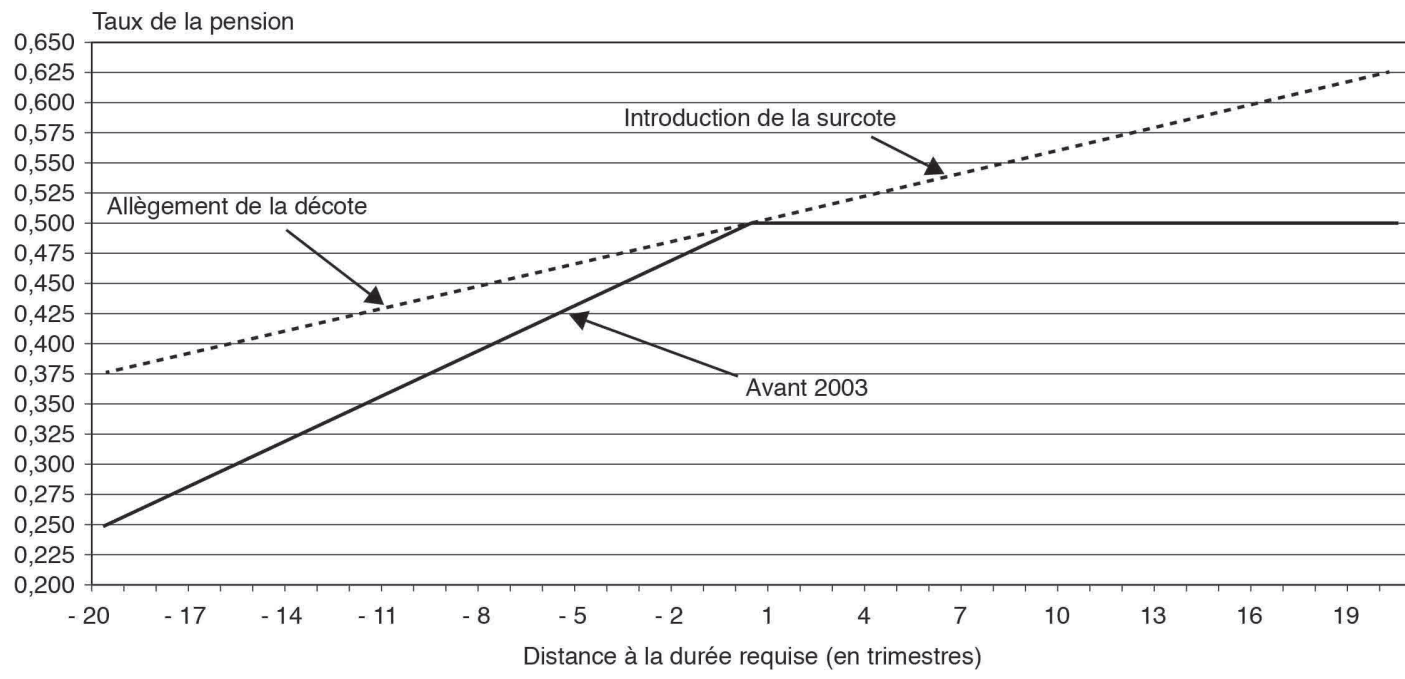

Lecture : avant la réforme des retraites de 2003, le taux de la pension est plafonné à $50 \%$ dès que la durée d'assurance requise est atteinte. À terme, lorsque la surcote s'appliquera intégralement comme le prévoit la réglementation de 2009, le taux de la pension augmentera de $5 \%$ pour chaque année supplémentaire travaillée au-delà de la durée requise.

Champ : assurés du régime général. 
de trimestres validés au titre du dispositif (cf. tableau 1). Ce dernier passe ainsi d'environ quatre trimestres en 2005 à près de sept trimestres en 2009, soit une augmentation de plus de $75 \%$ en quatre ans de la durée validée au titre de la surcote.

Ces informations sont toutefois difficiles à interpréter. Elles peuvent en effet laisser penser à un recours accru au dispositif alors qu'elles illustrent également sa montée en charge. L'objectif de la surcote étant d'inciter au décalage du départ en retraite, il est logique que ceux qui en bénéficient soient de plus en plus nombreux au cours des premières années qui suivent sa création. Il en est de même pour la durée de la surcote. La première année, celle-ci est nécessairement courte dans la mesure où elle

\section{Tableau 1 \\ Durée moyenne de la surcote entre 2005 et 2009}

\begin{tabular}{|l|c|c|c|}
\hline & Hommes & Femmes & Ensemble \\
\hline $\mathbf{2 0 0 5}$ & 3,9 & 3,9 & 3,9 \\
\hline $\mathbf{2 0 0 6}$ & 5,2 & 5,1 & 5,2 \\
\hline $\mathbf{2 0 0 7}$ & 6,1 & 5,9 & 6,0 \\
\hline $\mathbf{2 0 0 8}$ & 6,4 & 6,2 & 6,3 \\
\hline $\mathbf{2 0 0 9}$ & 7,0 & 6,5 & 6,8 \\
\hline
\end{tabular}

Lecture : les bénéficiaires de la surcote durant l'année 2006 ont une durée moyenne de surcote de 5,2 trimestres.

Champ : bénéficiaires de la surcote au régime général entre 2005 et 2009.

Source : flux exhaustif des nouveaux retraités du Régime Général (RG), SNSP (Infocentre). ne peut avoir débuté qu'au premier trimestre de la même année. Ainsi, les assurés qui liquident une pension avec surcote en 2005 ne peuvent logiquement avoir cotisé plus de sept trimestres à ce titre, alors que ceux de l'année 2009 peuvent avoir «surcoté » jusqu'à vingt-trois trimestres.

Par ailleurs, bien que le nombre de bénéficiaires de la surcote et le nombre moyen de trimestres validés au titre du dispositif aient fortement augmenté entre 2004 et 2009, l'âge moyen de départ en retraite des « surcoteurs » est resté relativement stable, autour de 62,7 ans (tableau 2).

Jusqu'à présent, l'impact de l'augmentation du nombre de surcoteurs sur l'âge moyen de départ en retraite de l'ensemble des retraités de droit

\section{Tableau 2 \\ Âge moyen de liquidation des droits à la retraite des bénéficiaires de la surcote}

\begin{tabular}{|l|c|c|c|}
\hline & Hommes & Femmes & Ensemble \\
\hline $\mathbf{2 0 0 4}$ & 62,5 & 62,6 & 62,5 \\
\hline $\mathbf{2 0 0 5}$ & 62,8 & 62,8 & 62,8 \\
\hline $\mathbf{2 0 0 6}$ & 62,8 & 62,8 & 62,8 \\
\hline $\mathbf{2 0 0 7}$ & 62,8 & 62,5 & 62,7 \\
\hline $\mathbf{2 0 0 8}$ & 62,8 & 62,5 & 62,6 \\
\hline $\mathbf{2 0 0 9}$ & 62,9 & 62,5 & 62,7 \\
\hline
\end{tabular}

Lecture : en 2009, l'âge moyen de liquidation des droits à la retraite des bénéficiaires de la surcote s'élève à 62,7 ans.

Champ : bénéficiaires de la surcote au régime général entre 2005 et 2009.

Source : flux exhaustif des nouveaux retraités du Régime Général (RG), SNSP (Infocentre).
Encadré 1 (suite)

entre le $1^{\text {er }}$ janvier 2004 et le 31 décembre 2008, les trois taux précédents continuent de s'appliquer.

L'objectif explicite de la surcote est d'encourager financièrement les assurés à liquider leur retraite plus tardivement tout en se maintenant en emploi. Ce dispositif a ainsi été créé afin de «corriger" la formule de calcul de la pension qui plafonnait jusqu'en 2003 le taux de la pension à $50 \%$. Les individus qui travaillaient après avoir obtenu la durée d'assurance requise ne voyaient en effet aucune amélioration de leur pension au régime général. Ces derniers ne pouvaient voir leur pension du régime général croître que par l'augmentation potentielle du salaire annuel moyen (prise en compte de salaires plus récents). Par contre, des droits supplémentaires étaient acquis dans les régimes complémentaires (augmentation du nombre de points acquis). Avant la réforme de 2003, les assurés qui liquidaient une pension avec une durée d'assurance validée inférieure à celle requise (distance néga- tive dans le graphique) bénéficiaient d'une forte décote alors que ceux qui disposaient d'une durée supérieure (distance supérieure à 0 dans le graphique) bénéficiaient d'une pension plafonnée à $50 \%$ de la moyenne de leurs meilleurs salaires, quelle que soit leur durée d'assurance. Cela pouvait créer une désincitation à poursuivre une activité au-delà de la durée d'assurance requise dans la mesure où celle-ci n'était pas, dans les régimes de base, valorisée financièrement. La réforme de 2003 a modifié le profil d'évolution du taux de la pension selon le nombre de trimestres validés. Les assurés disposant d'une durée inférieure à celle requise devraient être moins pénalisés financièrement avec l'allègement prévu de la décote. A contrario, ceux qui ont une durée d'assurance supérieure à celle requise peuvent améliorer leurs droits à pension avec la création de la surcote. À terme, à partir du vingtième trimestre manquant pour atteindre la durée requise, les droits à pension devraient augmenter linéairement avec le nombre de trimestres validés. 
direct du régime général n'est d'ailleurs pas visible. Ce dernier n'a en effet pas augmenté depuis l'entrée en vigueur de la surcote en 2004 (graphique II). Il a au contraire diminué entre 2003 et 2004 puis augmenté entre 2008 et 2009 avec la mise en place puis le durcissement du dispositif de retraites anticipées « carrières longues », qui permet à certains assurés de partir en retraite avant l'âge de 60 ans.

L'annulation de l'effet de ce dispositif ne permet pas de constater de réelle hausse de l'âge de départ moyen de départ en retraite potentiellement imputable à la surcote, même si l'on peut observer une légère augmentation entre 2008 et 2009. La déformation de la structure démographique des départs à la retraite liée au phénomène du « papy-boom » explique également certaines des évolutions constatées sur l'âge moyen de liquidation des droits à la retraite (3) (Benallah et Mette, 2009).

En résumé, bien qu'elle concerne une part de plus en plus importante des nouveaux retraités depuis 2004, la surcote n'a pour le moment aucun impact visible, au niveau macro, sur l'âge de départ en retraite. Les effets conjoncturels (montée en charge du dispositif, différence de taille des générations) ne permettent pas de distinguer un tel impact. À ces éléments conjonc- turels s'ajoute un potentiel effet d'aubaine, dans la mesure où certains assurés poursuivaient une activité après avoir obtenu le taux plein avant la création de la surcote. D'après Albert et al. (2008), 7 \% des assurés étaient concernés par cette pratique avant la réforme des retraites de 2003. Pour isoler les effets structurels de la surcote, il est donc nécessaire d'adopter une stratégie d'évaluation qui garantisse de neutraliser les effets conjoncturels et d'aubaine. Seule une analyse par génération, en distinguant les générations qui sont concernées par la surcote de celles qui ne le sont pas, permettrait de mesurer les modifications de comportements de départ en retraite imputables à la mise en place de la surcote. Nous proposons une telle analyse dans la suite de l'article.

\section{Mise en ouvre de l'évaluation des effets de la surcote}

Nous mettons en œuvre une évaluation des effets de la surcote sur les comportements de départ

\begin{abstract}
3. Entre 2004 et 2009, la structure démographique des flux s'est fortement modifiée sous l'effet du départ en retraite des premières générations nombreuses du baby-boom. Par exemple, la taille de la génération 1948, celle qui part à 60 ans en 2008, est plus importante que celle de la génération 1943 (effet " babyboom »), qui part à 65 ans la même année.
\end{abstract}

\section{Graphique II \\ Âge moyen de liquidation des droits à la retraite au régime général et âge moyen hors retraites anticipées entre 2004 et 2009}

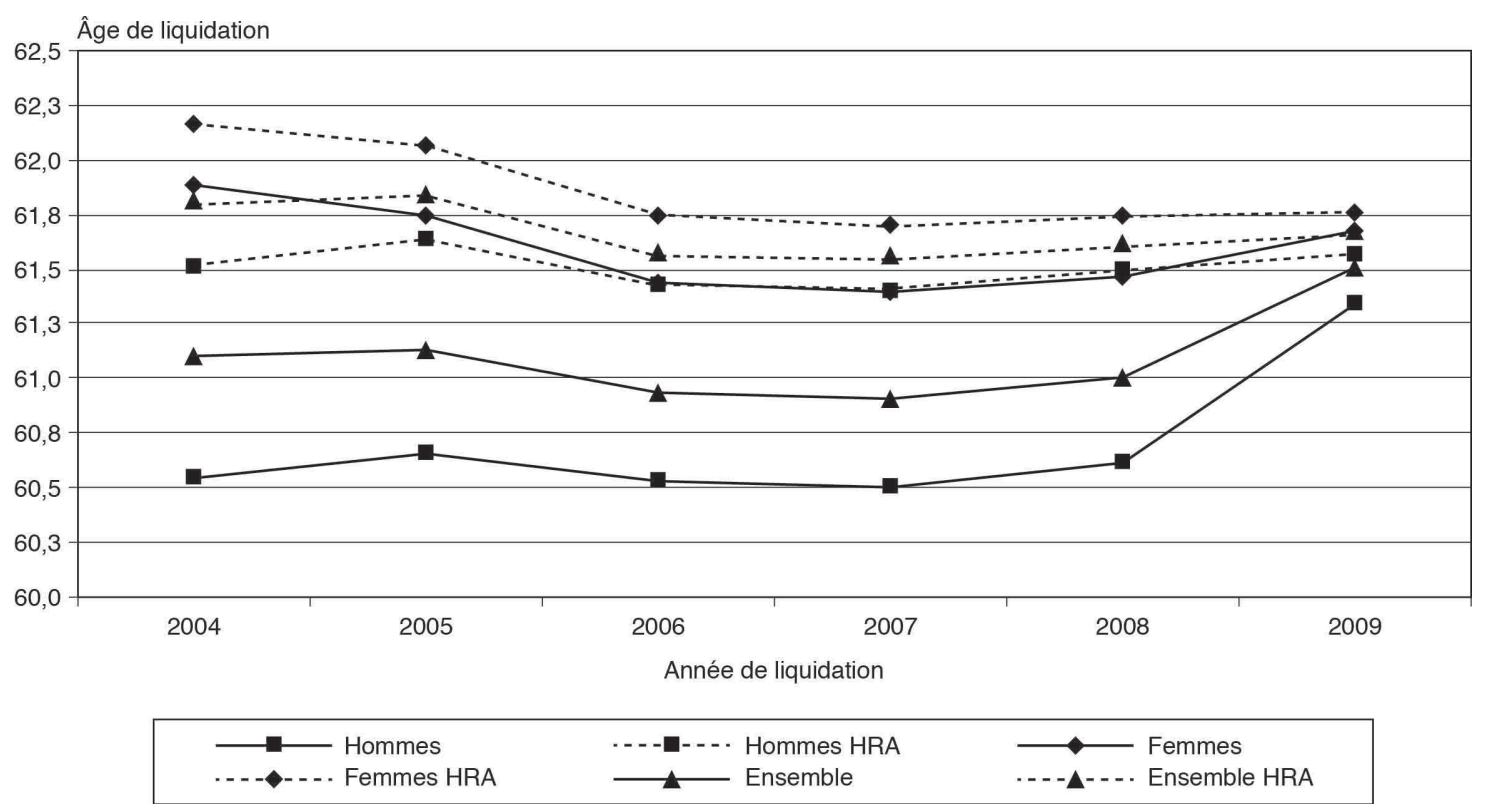

Lecture : les assurés ayant liquidé leur retraite en 2005 l'ont fait en moyenne à 61,1 ans. HRA : hors retraites anticipées.

Champ : assurés du régime général ayant liquidé leur retraite entre 2004 et 2009.

Source : flux exhaustif des nouveaux retraités du Régime Général (RG), SNSP (Infocentre). 
en retraite en mobilisant la méthode d'évaluation par appariement (cf. encadré 2). Pour cela, il est nécessaire d'identifier les individus pour lesquels le recours à la surcote est possible dès 60 ans et ceux qui n'ont aucune possibilité d'y recourir alors qu'ils remplissent les conditions d'éligibilité au dispositif. Il existe deux sources de limitation d'accès à la surcote. La première est la durée validée à l'âge de 60 ans. En s'appliquant aux trimestres " cotisés » au-delà de la durée requise pour bénéficier d'une retraite sans pénalité, la surcote est limitée aux assurés disposant d'une durée validée importante. Dans la mesure où elle peut relever de l'assuré luimême et potentiellement de son désir de recourir à la surcote, cette source de limitation ne peut être considérée comme un choc exogène. On ne peut donc pas s'en servir pour évaluer l'impact de la surcote sur les comportements de départ en retraite.

La seconde source de limitation est la date de mise en place du dispositif. En s'appliquant uniquement aux trimestres cotisés à partir du $1^{\text {er }}$ janvier 2004 et par conséquent aux pensions de retraite prenant effet à compter du $1^{\text {er }}$ avril 2004, la surcote est de facto limitée aux personnes nées à partir de 1939. Ces dernières ont en effet moins de 65 ans au moment où la surcote est mise en place. Elles ont donc la possibilité de poursuivre leur activité, au moins jusqu'à 65 ans, pour pouvoir en bénéficier. Les assurés des générations précédentes avaient en revanche déjà atteint l'âge de 65 ans en 2004. Ils avaient, dans l'ensemble, déjà pris leur retraite (4). Il est donc possible d'écrire, sans trahir le principe de non-exclusion par l'année de naissance à l'origine du dispositif, que la surcote s'applique en réalité uniquement aux personnes nées après 1938, autrement dit à celles qui avaient au plus 65 ans au cours de l'année 2004. Cette seconde limitation d'accès relève d'une décision du législateur et est, à ce titre, totalement indépendante de la volonté de l'assuré de recourir à la surcote. En ce sens, elle assure une variabilité interindividuelle totalement exogène. On peut donc utiliser la date d'entrée en vigueur de la surcote comme moyen de séparer les individus en deux sous-populations : une première regroupe les individus ayant accès à la surcote dès leur soixantième anniversaire, indépendamment de leur volonté d'y avoir recours ; une seconde sous-population est construite en retenant les individus qui ne peuvent pas recourir au dispositif même s'ils souhaitent le faire. Aussi, compte tenu de la date d'entrée en vigueur du dispositif, les deux groupes sont construits de la manière suivante : d'un côté, en 2004, la géné- ration 1944 atteignait l'âge de 60 ans et était ainsi la génération la plus jeune pouvant prétendre à une pension surcotée en se maintenant en activité et en repoussant son départ en retraite. On considère ainsi cette génération comme « le groupe de traitement ». De l'autre côté, la génération 1938 atteignait 66 ans en 2004 ce qui signifie qu'elle n'a pas pu bénéficier de la surcote en prolongeant son activité et en reculant son âge de départ en retraite. Si certains assurés de cette génération ont prolongé leur activité après 60 ans et au-delà de la durée requise pour le taux plein, c'est donc sans considération de la surcote. Par conséquent, la génération 1938 est considérée comme le « groupe de contrôle » de notre évaluation, autrement dit notre population « non traitée ».

Parmi les générations 1944 et 1938, tous les assurés ne sont toutefois pas éligibles à la surcote. C'est le cas des individus qui arrivent à l'âge de 60 ans avec un nombre de trimestres faible. Il nous faut donc restreindre notre analyse aux potentiels « surcoteurs », c'est-à-dire aux individus qui sont éligibles au dispositif en termes de durée d'assurance validée. Une façon de faire est de retirer de l'analyse tous les assurés qui arrivent à l'âge de 60 ans en ne disposant pas du nombre de trimestres requis. Cela revient à ne retenir que les individus qui disposent, à l'âge de 60 ans, de la durée requise pour bénéficier d'une retraite au taux plein. L'inconvénient de ce critère est qu'il est basé sur une condition de durée nécessaire pour le taux plein. Or, avec l'allongement de la durée d'assurance requise prévu par les réformes de 1993 et de 2003, cette durée n'est pas identique pour les générations 1938 et 1944 . Les individus nés en 1938 doivent en effet justifier de 155 trimestres pour prétendre à une retraite au taux plein alors que ceux nés en 1944 doivent en valider 160. Ce critère crée donc une source de différenciation entre les deux sous-populations, autre que celle induite par l'éligibilité au dispositif de la surcote.

La méthode d'évaluation que nous mobilisons n'étant valable que si les deux sous-populations ne sont pas différentiables, au critère d'éligibilité à la surcote près, les résultats des estimations basées sur ces critères de sélection risqueraient d'être biaisés. Pour annuler cette seconde source de différenciation, nous ne retenons que les assurés qui ont validé au moins 160 trimestres l'année de leur $60^{\text {ème }}$ anniversaire (hors

4. D'après les données de l'échantillon au $20^{\text {ème }}$ des assurés de la Cnav, près de $98 \%$ des retraités de la génération 1938 avaient déjà pris leur retraite en 2004, l'année de leur 66 ${ }^{\text {ème }}$ anniversaire. 
Encadré 2

\section{LA MÉTHODE D'ÉVALUATION}

Nous cherchons à mesurer l'impact de la surcote sur les comportements de départ à la retraite depuis 2004 en comparant les décisions de départ en retraite effectivement observées à celles qui auraient potentiellement été prises, en l'absence du dispositif de la surcote. Pour cela, nous partons du cadre théorique proposé par Rubin (1974) qui permet, sous certaines conditions, d'établir un lien causal entre deux évènements à partir de la notion centrale de « résultat potentiel ». La situation de l'individu $i$ vis-à-vis de la surcote, notée $S_{i}$, ne peut en effet être observée qu'une fois. Aussi, soit l'individu peut bénéficier de la surcote s'il recule son départ en retraite (dans ce cas, nous considérons que $S_{i}$ vaut 1 ), soit il ne peut y avoir accès même s'il retarde son départ (et $S_{i}$ vaut alors 0 ). De la même façon, chaque individu ne prend sa retraite qu'une fois, ce qui implique que, pour chaque individu, on ne peut observer qu'un seul âge de départ en retraite, noté $R$. Cela empêche d'établir l'effet causal de la surcote au niveau individuel. Par contre, il est possible, sous certaines hypothèses, de le mesurer au niveau moyen. Nous nous intéressons plus particulièrement à l'effet moyen du dispositif sur la population concernée par la réforme ou, en d'autres termes, si l'on considère la mise en place de la réforme de 2003 comme un traitement " administré » à des individus, à l'effet moyen du traitement sur la population traitée (Average Treatment effect on the Treated - ATT). Pour déduire un tel effet, nous faisons l'hypothèse que les comportements de retraite des générations concernées par la surcote, dans le cas où elles ne l'auraient pas été, peuvent être déduits de ceux observés pour les générations qui ne sont pas concernées par la surcote, ce qui revient à écrire :

$$
E\left[R_{i}(0) \mid \mathrm{S}_{i}=1\right]=E\left[R_{i}(0) \mid S_{i}=0\right]
$$

Ces dernières sont ainsi supposées être l'exact reflet des individus composant les générations concernées par la surcote dans le cas théorique où ils ne l'auraient pas été. Pour que cette hypothèse soit réaliste, il est indispensable que les individus concernés par la surcote et ceux qui ne le sont pas ne présentent pas de différences (autre que l'éligibilité à la surcote) susceptibles de faire diverger leurs décisions de retraite. Or, la réforme des retraites de 2003 ne relève pas d'une mise en œuvre par échantillonnage aléatoire (la législation issue des réformes s'applique en effet principalement en fonction de l'année de naissance), et il peut par conséquent exister des différences entre les générations touchées par la réforme et celles qui ne le sont pas.

Il est alors important de limiter la comparaison aux individus " comparables », c'est-à-dire disposant des mêmes caractéristiques observables $X$, ce qui implique que la condition d'identification (1) s'écrit désormais :

$$
E\left[R_{i}(0) \mid S_{i}=1, X_{i}\right]=E\left[R_{i}(0) \mid S_{i}=0, X_{i}\right]
$$

Si une telle comparaison est mise en œuvre, la réforme est alors considérée comme distribuée aléatoirement « conditionnellement aux caractéristiques observa- bles ». Cela signifie que, compte tenu des caractéristiques $X_{i}$ différentes de l'éligibilité à la surcote, les comportements de départ en retraite des individus touchés par la surcote auraient été identiques, en l'absence de mise en place du dispositif, à ceux des individus qui ne sont pas concernés par la surcote.

Réaliser un appariement entre les deux sous-populations sur les caractéristiques observables revient à trouver pour chaque individu concerné par la réforme son « équivalent » non concerné compte tenu des caractéristiques retenues. S'agissant de l'évaluation du dispositif de la surcote, le critère différenciant les personnes concernées et celles qui ne le sont pas est l'année de naissance. Ce critère renvoie à des parcours sur le marché du travail différents. Parvenir à identifier des individus qui se ressemblent en tous points, c'est-à-dire qui ont exactement le même parcours professionnel, est compliqué. Le nombre des caractéristiques est en effet élevé et il est difficile de réaliser un appariement sur l'ensemble de ces caractéristiques. Une solution proposée par Rosenbaum et Rubin (1983) est d'apparier sur le score de propension $e(X)$, défini ici comme la probabilité, pour un individu $i$ possédant les caractéristiques $X$, d'être concerné par le dispositif de la surcote :

$$
e\left(X_{i}\right)=\operatorname{Pr}\left(S_{i}=1 \mid X_{i}\right)
$$

Les auteurs montrent en effet que lorsque la variable d'intérêt est indépendante de l'éligibilité au traitement conditionnellement aux variables observables, elle l'est également conditionnellement au score de propension. Ce dernier peut ainsi constituer un résumé des caractéristiques observables d'un individu, une représentation unidimensionnelle. II est alors possible d'écrire la condition d'identification de la manière suivante:

$$
E\left[R_{i}(0) \mid \mathrm{S}_{i}=1, e\left(X_{i}\right)\right]=E\left[R_{i}(0) \mid S_{i}=0, e\left(X_{i}\right)\right]
$$

Cette condition n'est équivalente à l'égalité (2) que si, à l'issue de l'appariement par le score de propension, la variable de traitement $S$ ne dépend plus de $X$ (propriété d'équilibrage du score de propension). Sous ces hypothèses, l'effet moyen de la surcote sur la population concernée par la réforme est évalué de la manière suivante :

$$
\begin{array}{r}
\Delta_{A T T}=E_{e\left(X_{i}\right) \mid S_{i}=1}\left\{E\left[R_{i}(1) \mid S_{i}=1, e\left(X_{i}\right)\right]-E\left[R_{i}(0) \mid\right.\right. \\
\left.\left.S_{i}=0, e\left(X_{i}\right)\right]\right\}
\end{array}
$$

Une telle méthode d'évaluation présente de nombreux avantages en comparaison des méthodes traditionnelles de régression paramétrique. D'une part, elle permet de prendre en compte des caractéristiques observables dans l'estimation sans imposer a priori de relation fonctionnelle entre ces caractéristiques et la décision de départ en retraite et limite l'évaluation à des individus comparables. D'autre part, en mesurant un effet de la surcote pour chaque individu concerné par le dispositif, elle permet de tenir compte de l'effet différencié de la surcote suivant les individus. 
majoration de durée d'assurance pour les femmes). Cette solution permet ainsi de placer au même point de départ tous les individus soumis à l'évaluation, quel que soit le groupe auquel ils appartiennent (groupe de traitement ou de contrôle) et sans considération des différences de durée requise pour obtenir le taux plein entre les deux générations. Les individus de la génération 1938 et de la génération 1944 ont ainsi, au départ, une propension à repousser leur départ à la retraite strictement identique (les modalités de construction des deux groupes sont résumées dans le tableau 3).

\section{L'échantillon des assurés de la Cnav}

Pour procéder à une telle dichotomie, il est nécessaire de disposer de données longitudinales qui permettent de distinguer les deux cohortes d'assurés mais également de connaître la durée que les individus appartenant à chacune de ces cohortes ont validée avant de pouvoir partir en retraite. L'échantillon des assurés de la Cnav est doté de telles caractéristiques. Il s'agit en effet d'un échantillon (au vingtième) représentatif des assurés du régime général d'assurance-vieillesse. Ces derniers sont aussi bien des cotisants, c'est-à-dire des personnes qui ont au moins une validation au régime général d'assurance-vieillesse mais qui n'ont pas encore liquidé leur retraite, que des retraités. Il est enrichi tous les deux ans des nouveaux assurés du régime général et des données actualisées pour ce qui est des assurés qui sont initialement présents dans l'échantillon, avec une mise à jour semestrielle des départs en retraite.

En plus de permettre le suivi de cohortes de manière persistante dans le temps, de leur première validation à leur départ en retraite, les données de l'échantillon de la Cnav apportent des informations très fines sur l'assuré, particulièrement sur le déroulement de sa carrière. Pour mieux comprendre la richesse de ces données, il est utile de rappeler la manière dont les fichiers de la Cnav sont constitués et le type d'information que cette dernière recueille. Par définition, les données recueillies par la Cnav ont pour but de déterminer les droits à la retraite auxquels peuvent prétendre ses assurés. Ces droits dépendent de nombreux paramètres qui ont trait à la fois à des caractéristiques personnelles (la date de naissance par exemple) ainsi qu'à des éléments de carrière. Les fichiers de gestion de la Cnav, appelés les « Référentiels Nationaux », sont ainsi principalement alimentés par trois sources correspondant aux trois types d'informations recueillies : les informations permettant d'établir une immatriculation qui sont principalement alimentées par les mairies ; les informations salariales qui émanent des employeurs par le biais des déclarations annuelles de données sociales ; et les informations complémentaires de carrière qui sont transmises par les différents partenaires de la sphère sociale (UNEDIC, CPAM, CAF, autres régimes de retraite, etc.).

Nous disposons donc, pour notre évaluation, d'un résumé de l'ensemble des informations recueillies par la Cnav sur un échantillon au vingtième de l'exhaustif des assurés du régime général d'assurance-vieillesse. Nous avons ainsi un échantillon très large d'individus pour lesquels nous disposons d'informations administratives assez fines et qui nous permettent notamment de retracer l'ensemble de leur carrière, jusqu'à leur départ en retraite. Cette source de données est particulièrement précieuse compte tenu de la manière dont nous sélectionnons les individus appartenant au groupe de traitement et ceux appartenant au groupe de contrôle. Il nous faut en effet disposer de leur année de naissance mais aussi connaître l'état de leur carrière à leur $60^{\text {ème }}$ anniversaire pour ne sélectionner, aussi bien pour le groupe de traitement que pour celui de contrôle, que les assurés ayant validé au moins 160 trimestres à cette date.

Pour mettre en œuvre l'évaluation de la surcote, il nous faut par ailleurs rendre les générations 1938 et 1944 comparables. Pour ce faire, nous retenons, pour le calcul du score de propension, une série de variables résumant le parcours de l'assuré sur le marché du travail ainsi que des variables sociodémographiques et une information sur le revenu (cf. tableau A en annexe). Le parcours de l'assuré est découpé en deux parties. Du premier enregistrement de carrière à l'âge de

Tableau 3

Construction du groupe « traité " et du groupe de contrôle

\begin{tabular}{|l|l|}
\hline \multicolumn{1}{|c|}{ Groupe traité } & \multicolumn{1}{c|}{ Groupe de contrôle } \\
\hline $\begin{array}{l}\text { Assurés nés en 1944 ayant validé au moins 160 trimestres } \\
\text { l'année de leurs } 60 \text { ans } \\
\text { (hors majoration de durée d'assurance) }\end{array}$ & $\begin{array}{l}\text { Assurés nés en 1938 ayant validé au moins 160 trimestres } \\
\text { l'année de leurs 60 ans } \\
\text { (hors majoration de durée d'assurance) }\end{array}$ \\
\hline
\end{tabular}

Source : auteure. 
55 ans, nous retenons trois variables : la part de la carrière (au sens strict, c'est-à-dire les trimestres cotisés) qui relève du régime général (autrement dit qui correspond au statut de salarié du privé) ; la part de l'aléa chômage dans la carrière ; et la part de la maladie dans les trimestres validés. Nous prêtons ensuite une attention particulière à ce qui se passe entre 55 et 60 ans. Il s'agit en effet d'une phase critique dans la carrière des individus, susceptibles d'affecter leurs choix de départ en retraite (Magnac et al., 2006). Nous prenons ainsi en compte le nombre de trimestres de chômage et de maladie dans la période qui précède le départ à la retraite. Nous ajoutons enfin, pour résumer la carrière, le nombre de trimestres validés à 60 ans (qui ne peut être inférieur à 160) qui reflète la longueur de la carrière, et la moyenne des dix meilleures années de salaires enregistrés jusqu'à l'âge de 60 ans (5).

Le choix des caractéristiques observables intégrées dans le calcul du score de propension est une étape délicate de la mise en œuvre des modèles d'appariement. En nous focalisant sur le déroulement de la carrière, nous faisons implicitement l'hypothèse que la principale source de différenciation entre la génération 1938 et la génération 1944, hors surcote, est le parcours sur le marché du travail. Ce choix tient à la fois à des constatations empiriques (entrée dans la vie active plus tardive, carrières caractérisées par davantage d'aléas et de mobilités, etc.) mais également à la disponibilité des données. Comme nous l'avons dit plus haut, les informations que nous mobilisons ont pour but de déterminer les droits à la retraite auxquels peuvent prétendre les assurés de la Cnav. Pour cette raison, certaines informations tels la situation familiale, la catégorie socioprofessionnelle ou encore le secteur d'activité sont absentes des fichiers de gestion de la Cnav, et ne sont donc pas pris en compte dans le calcul du score (6). Cela peut poser problème si pour chacune de ces informations, des évolutions importantes ont pu être constatées entre la génération 1938 et la génération 1944, et que ces dernières ont une influence sur les comportements de retraite. Rien n'indique que de telles évolutions aient eu lieu.

La surcote aurait provoqué une augmentation de deux mois de l'âge de liquidation des droits à la retraite et de $16 \%$ de la probabilité de rester en emploi après 60 ans

La surcote a pour objectif d'inciter les individus à retarder leur départ à la retraite en se main- tenant en emploi. Pour étudier son impact sur les comportements de départ en retraite, deux grandeurs présentent un intérêt : l'âge de liquidation des droits à la retraite et la durée cotisée au-delà de celle requise après 60 ans. En ce qui concerne l'âge de liquidation, tous les individus de la génération 1944 n’ont pas encore fait valoir leurs droits à la retraite. Ils ont en effet atteint l'âge de 65 ans en 2009 et certains d'entre eux liquideront après cet âge. Nous avons donc plafonné à 65 ans l'âge de liquidation des droits à la retraite pour la génération 1938 et 1944 . La probabilité d'être en emploi après 60 ans est quant à elle approximée par celle d'avoir cotisé au moins un trimestre après 60 ans. Les individus sélectionnés dans le cadre de notre étude ayant déjà validé au moins 160 trimestres à 60 ans, la probabilité de cotiser des trimestres supplémentaires après 60 ans est donc susceptible d'augmenter sous l'effet incitatif de la surcote.

Compte tenu du nombre important d'observations disponibles, nous avons procédé à un appariement sur les deux plus proches voisins en termes de score de propension à l'aide du module psmatch 2 du logiciel STATA (Leuven et Sianesi, 2003). L'appariement a également été réalisé à partir d'une fonction noyau - Kernel normal matching - sans modification substantielle des résultats. Afin que les voisins les plus proches ne soient pas trop éloignés, nous supprimons de l'estimation les individus appartenant au groupe de traitement dont le score de propension excède le score maximal du groupe de contrôle. Nous supprimons également les individus du groupe de traitement pour lesquels les plus faibles densités du score de propension dans le groupe de contrôle sont enregistrées et imposons une distance maximale de $1 \%$ entre les individus traités et leurs homologues non traités. La fenêtre de support commun des scores de propension reste importante, comprise entre 0,16 et 0,97 , et la part d'individus traités exclus des estimations est relativement limitée (environ $2 \%$ ). Enfin, nous avons procédé à une série de tests (calculs des biais standardisés et

5. En ce qui concerne la moyenne des meilleures années de salaire, nous la calculons à partir des salaires plafonnés. Cela est problématique dans la mesure où un même salaire (au plafond) pour deux individus peut en réalité cacher des situations très contrastées en termes de revenus.

6. C'est pour cette même raison qu'il nous est impossible de traiter d'un éventuel biais de sélection lié à des caractéristiques inobservables. Les méthodes traitant cette problématique requièrent en effet de disposer d'une ou plusieurs variables instrumentales expliquant la probabilité d'être éligible à la surcote sans contribuer directement à expliquer les décisions de retraite. La prise en compte d'un biais de sélection lié à des variables inobservables pourrait être l'objet de travaux futurs, à condition de disposer de données complémentaires issues d'autres sources que les fichiers de la Cnav. 
pseudo- $\mathrm{R}^{2}$ avec la commande pstest du module psmatch2) afin de vérifier la propriété d'équilibrage du score de propension. Pour l'ensemble des résultats présentés, la propriété d'équilibrage est vérifiée : l'ajustement par l'appariement équilibre les distributions des variables entre les groupes traités et leurs contrefactuels.

La surcote semble avoir eu un impact sur les comportements de départ en retraite (cf. tableau 4). On peut y observer que l'âge moyen de départ en retraite de la génération 1944 est de 60,56 ans. Il aurait été de 60,37 ans sans la mise en place de la surcote (estimation du contrefactuel). Ainsi, les assurés nés en 1944 seraient partis en retraite deux mois plus tôt s'ils n'avaient pas pu bénéficier du dispositif.

On retrouve des résultats comparables concernant la probabilité de poursuivre une activité après 60 ans et au-delà de 160 trimestres : $53 \%$ des assurés nés en 1944 et disposant d'au moins 160 trimestres à 60 ans ont poursuivi une activité après 60 ans. En l'absence de la surcote, ils auraient été $45 \%$ à en faire de même. Cela représenterait une amélioration de la probabilité de poursuivre une activité après 60 ans et audelà de 160 trimestres imputable à la mise en place de la surcote de plus de $16,5 \%$.

\section{Tableau 4}

Effet de la surcote sur l'âge de liquidation des droits à la retraite et la probabilité de prolonger son activité après 60 ans au-delà de la durée requise

\begin{tabular}{|c|c|c|c|}
\hline & $\begin{array}{c}\text { Génération } \\
1944\end{array}$ & $\begin{array}{c}\text { Génération } \\
1938 \\
\text { (contrefactuel) }\end{array}$ & Différence \\
\hline \multicolumn{4}{|c|}{ Âge de liquidation des droits à la retraite } \\
\hline $\begin{array}{l}\text { Avant } \\
\text { appariement }\end{array}$ & 60,565 & 60,346 & $\begin{array}{l}0,218^{\cdots} \\
(0,018)\end{array}$ \\
\hline $\begin{array}{l}\text { Après } \\
\text { appariement }\end{array}$ & 60,559 & 60,370 & $\begin{array}{l}0,189 \cdots \\
(0,053)\end{array}$ \\
\hline \multicolumn{4}{|c|}{ Probabilité de prolonger son activité après 60 ans } \\
\hline $\begin{array}{l}\text { Avant } \\
\text { appariement }\end{array}$ & 0,532 & 0,380 & $\begin{array}{l}0,151 \ldots \\
(0,008)\end{array}$ \\
\hline $\begin{array}{l}\text { Après } \\
\text { appariement }\end{array}$ & 0,529 & 0,454 & $\begin{array}{l}0,075 \cdots \\
(0,027)\end{array}$ \\
\hline Effectifs & 9690 & 6707 & \\
\hline
\end{tabular}

Lecture : la différence est significative à : ${ }^{* *} 1 \%,{ }^{* *} 5 \%,{ }^{*} 10 \%$, écart-type entre parenthèses.

Champ : générations 1938 et 1944 ayant validé au moins 160 trimestres à 60 ans.

Source : échantillon au $20^{\text {ème }}$ des assurés de la Cnav.
L'impact observé de la surcote sur les comportements de départ en retraite (différence simple) est atténué par la prise en compte des différences de caractéristiques entre la génération 1938 et la génération 1944. Ainsi, sans correction de ces différences, l'impact de la surcote sur l'âge de liquidation des droits à la retraite et la probabilité de poursuivre une activité après 60 ans est plus important. Il est de l'ordre d'un trimestre pour l'âge moyen de liquidation et de 15 points de pourcentage pour la probabilité de cotiser des trimestres après 60 ans.

Nous disposons d'un nombre important d'observations, aussi bien pour le groupe de traitement que pour le groupe de contrôle. Il est de ce fait possible de procéder à l'estimation sur plusieurs sous-échantillons de l'effet causal de la surcote sur les comportements de départ en retraite. Nous pouvons de cette manière apprécier les impacts différenciés de la surcote selon la population concernée. Nous déclinons ainsi l'ensemble des résultats par genre, situation d'activité avant la retraite (les individus sont classés en deux groupes, selon qu'ils ont connu au moins une période de chômage entre 55 et 60 ans ou non), statut de pensionnés (monopensionnés ou polypensionnés) et durée validée à 60 ans. L'écart de comportement de départ en retraite imputable à la mise en place de la surcote est assez différencié selon les catégories de retraités (cf. graphiques III et IV). Il est plus important pour les hommes que pour les femmes. Il est de près d'un trimestre pour les hommes nés en 1944 et de l'ordre d'un mois pour les femmes nées la même année. Ces dernières sont par ailleurs déjà moins représentées dans l'évaluation que les hommes dans la mesure où leurs carrières sont moins linéaires ce qui réduit leur éligibilité à la surcote suivant le critère restrictif que nous avons retenu pour cette évaluation. $\mathrm{Ce}$ critère est particulièrement restrictif pour les femmes dans la mesure où nous n'intégrons pas les majorations de durée d'assurance qui permettraient à certaines d'entre elles d'atteindre 160 trimestres à 60 ans. L'impact de la surcote sur l'âge de départ en retraite semble également différencié selon la situation avant la retraite. Les individus n'ayant connu aucune période de chômage entre 55 et 60 ans partiraient en retraite plus tardivement de deux mois grâce à la surcote, contre un mois pour ceux ayant connu au moins un trimestre de chômage. L'âge moyen de départ en retraite des monopensionnés aurait également été davantage affecté par l'introduction de la surcote que celui des polypensionnés. Cette dernière aurait en effet provoqué un report de liquidation des droits à la retraite d'environ 
Graphique III

Âge moyen de départ en retraite des générations 1938 et 1944 après estimation par appariement

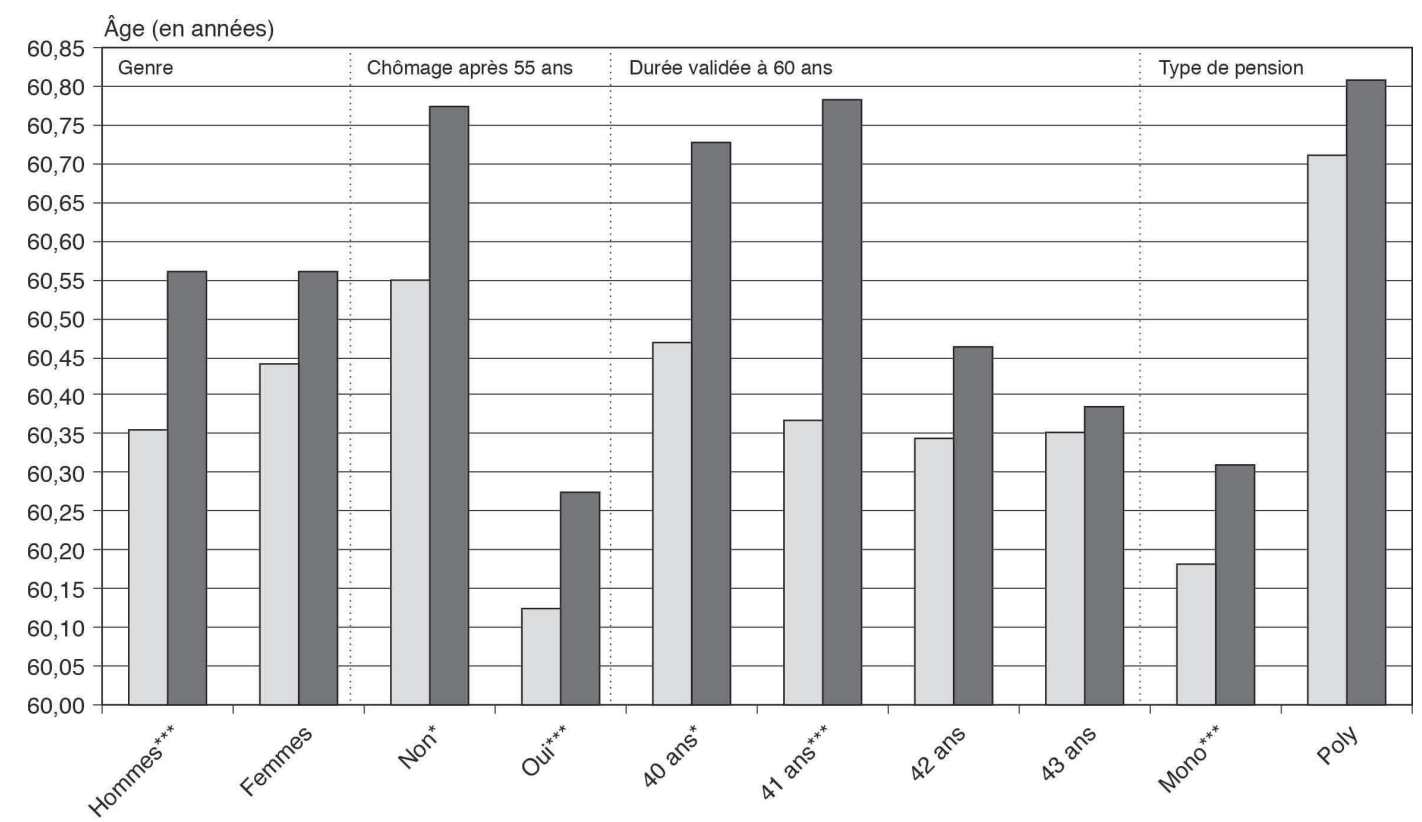

Type de pensionnés

$\square$ Contrefactuel $\square$ Génération 1944

Lecture : l'âge moyen de départ en retraite de la génération 1944 est de 60,56 ans. Il aurait été de 60,39 ans si la surcote n'avait pas été mise en place. L'estimateur est significatif à : *** $1 \%$, ${ }^{*} 5 \%$, * $10 \%$.

Champ : générations 1938 et 1944 ayant validé au moins 160 trimestres à 60 ans, respectivement 6707 et 9690 individus Source : échantillon au $20^{\mathrm{e} m e}$ des assurés de la Cnav.

Graphique IV

Probabilité de prolonger son activité au-delà de 60 ans des générations 1938 et 1944 après estimation par appariement

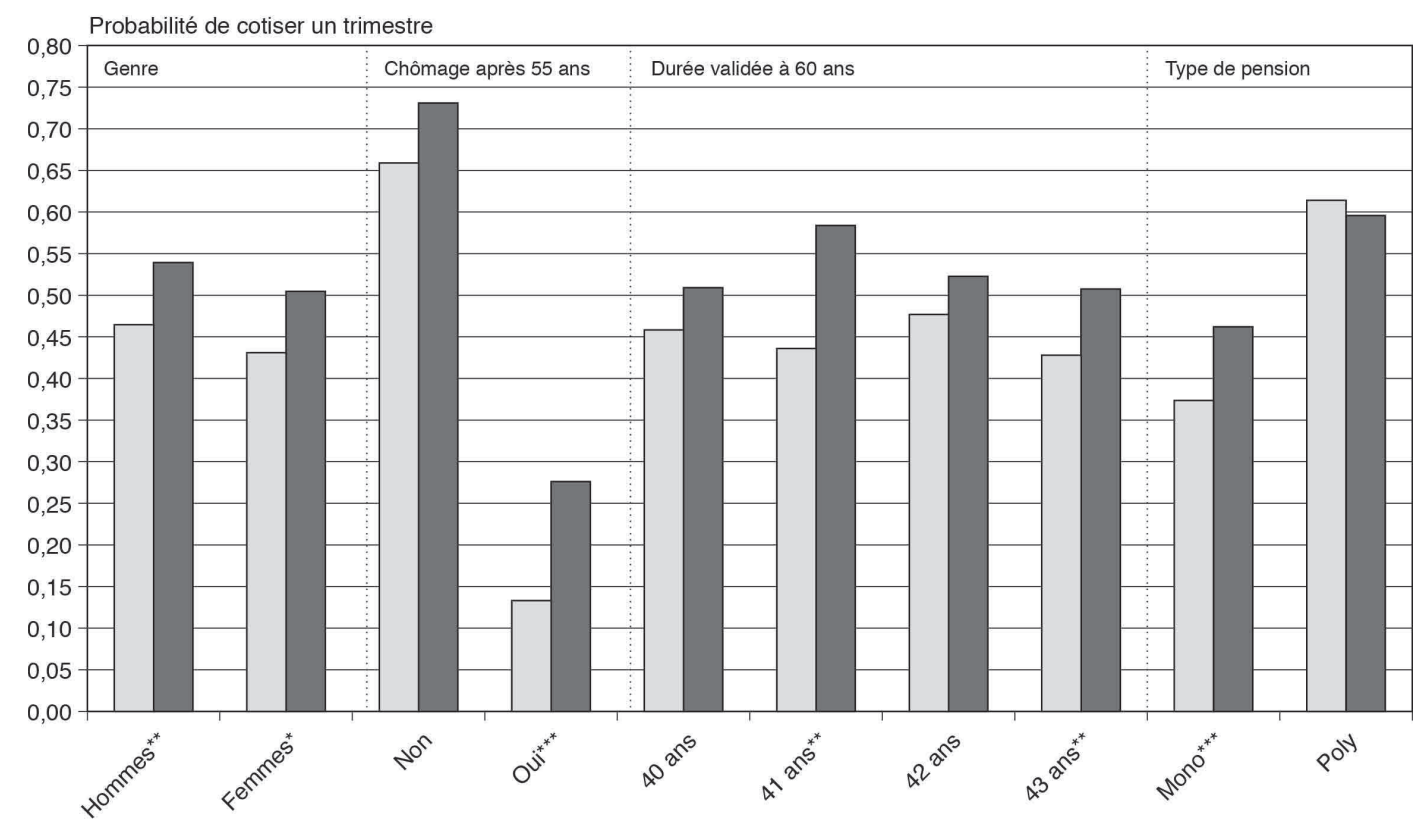

Type de pensionnés

$\square$ Contrefactuel $\square$ Génération 1944

Lecture : la probabilité de prolonger son activité au-delà de 60 ans des hommes de la génération 1944 est de 53,7\%. Elle aurait été de $46,8 \%$ si la surcote n'avait pas été mise en place. L'estimateur est significatif à : *** $1 \%,{ }^{* *} 5 \%$, $10 \%$.

Champ : générations 1938 et 1944 ayant validé au moins 160 trimestres à 60 ans, respectivement 6707 et 9690 individus. Source : échantillon au $20^{\text {ème }}$ des assurés de la Cnav. 
un mois chez les polypensionnés contre près de deux mois chez les monopensionnés. Il existe enfin des différences notables suivant la durée validée à 60 ans. La différence de comportement imputable à la mise en place de la surcote est croissante avec la durée validée à 60 ans jusqu'au seuil de 41 annuités puis décroissante. L'écart est le plus important pour les assurés ayant validé, à l'âge de 60 ans, entre 164 et 167 trimestres. Pour ces derniers, l'âge moyen de liquidation des droits à la retraite aurait été plus faible de cinq mois si la surcote n'avait pas été mise en place.

Concernant la probabilité de prolonger son activité après 60 ans, le graphique IV ne met pas en évidence de différence entre les hommes et les femmes : celle-ci augmenterait de 7 points de pourcentage sous l'effet de la surcote.

En revanche, des différences de sensibilité à la surcote suivant la situation d'emploi entre 55 et 60 ans, la catégorie de pensionnés et la durée validée à 60 ans subsistent. Les individus ayant connu du chômage entre 55 et 60 ans, qui avaient au départ une très faible propension à poursuivre une activité après 60 ans, voient leur probabilité de cotiser au moins un trimestre après cet âge doubler sous l'effet de la surcote, alors que cette dernière augmente de $10 \%$ pour ceux n'ayant pas connu de chômage en fin de carrière. Par ailleurs, les monopensionnés nés en 1944 ayant validé au moins 160 trimestres à 60 ans seraient particulièrement sensibles à la surcote puisque leur probabilité de poursuivre une activité après 60 ans semble avoir été améliorée de $23 \%$ avec la création du dispositif. Les polypensionnés seraient de leur côté moins disposés à poursuivre une activité. Ce résultat vient probablement de ce qu'une partie des assurés nés en 1944 n'ont pas encore liquidé leur retraite. Pour ces derniers, certains reports d'activité ne sont pas encore connus, précisément si ces derniers concernent les autres régimes de retraite (indépendants, fonctionnaires, etc.). Les polypensionnés de la génération 1944 pourraient donc être plus nombreux à avoir été concernés par des informations lacunaires quant à leur fin de carrière. Il est ainsi probable que l'on sous-estime l'impact de la surcote sur la probabilité de prolonger son activité au-delà de 60 ans pour cette catégorie d'assurés. On retrouve enfin une sensibilité à la surcote particulièrement forte pour les assurés disposant de 41 annuités validées à l'âge de 60 ans. Ces derniers verraient ainsi leur probabilité de poursuivre une activité augmenter de près de $33 \%$ sous l'effet de la surcote.

\section{Ces effets ne sont-ils imputables qu'à la création de la surcote ?}

Compte tenu de la stratégie d'identification mobilisée, l'impact de la surcote sur les comportements de départ en retraite que nous avons mesuré n'est valide qu'à condition que les groupes de traitement et contrefactuels ne soient pas différentiables, à l'éligibilité à la surcote près. Il est possible que des différences entre les deux générations n'aient pas été prises en compte dans le calcul du score de propension. Parmi ces différences, celles induites par le calcul des droits dans les régimes complémentaires peut biaiser les résultats si elle implique des pensions de retraite inégales entre les deux générations à carrière donnée. Une autre différence peut provenir de l'application de la réforme des retraites de 1993. Cette dernière a en effet modifié le nombre de salaires entrant dans le calcul du salaire annuel moyen (SAM - moyenne des meilleurs salaires permettant de calculer la pension de base du régime général). Ce dernier est ainsi passé de 10 à 25 entre les générations 1933 et 1953, à un rythme d'un salaire supplémentaire par génération. Cela signifie que les générations 1938 et 1944 voient leur pension de retraite assise sur un nombre de salaires différent, respectivement 15 et 21. Pour une même carrière, un individu né en 1944 peut par conséquent se voir attribuer une pension de retraite de base plus faible qu'un individu né en 1938, ce qui peut influencer sa décision de départ en retraite.

À ce titre, on peut se demander si les modifications de comportement que nous attribuons à la création de la surcote ne sont pas imputables à la modification du salaire annuel moyen imposée par la réforme des retraites de 1993. Un moyen de répondre à cette question est de limiter l'évaluation aux individus nés en 1944 qui disposent du même niveau de pension que celui qui leur aurait été affecté en application de la législation du SAM pour la génération 1938 (7). Cela est possible car, contrairement à la surcote qui concerne tous les futurs retraités de la génération 1944 qui disposent de la durée requise, l'augmentation du nombre de salaires inclus dans le SAM n'affecte pas systématiquement leur retraite. Il existe en effet de nombreux cas pour lesquels la modifi-

\footnotetext{
7. Une autre façon de faire serait de sélectionner, parmi les personnes nées en 1944, des assurés non concernés par la surcote et de les comparer à leurs homologues de la génération 1938 afin d'éliminer l'effet du SAM par une démarche de double différence. La réforme de 1993 a toutefois créé d'autres différences entre les individus des deux générations non éligibles à la surcote (différences de durée requise, de durée retenue pour la proratisation et de niveau de la décote) si bien qu'une telle stratégie serait inefficace pour neutraliser l'effet du SAM et donc isoler l'impact de la surcote.
} 
cation du SAM n'implique aucun effet sur le montant de la pension : carrière linéaire, ensemble des salaires au-dessus du salaire plafond de la Sécurité sociale, pension au niveau du minimum contributif ou du maximum des pensions, etc. À cela s'ajoutent les dispositions introduites par la réforme des retraites de 2003 qui visent à amortir les effets négatifs de la prise en compte d'un plus grand nombre de salaires dans le calcul de la pension (exclusion des salaires ne permettant pas de valider un trimestre et proratisation du nombre de salaires portés au compte pour les polypensionnés, pour une présentation détaillée de ces mesures voir Benallah, 2009). Nous avons donc calculé deux SAM pour les individus composant la génération 1944 : le premier suivant la législation de la génération 1938, l'autre suivant celle de la génération 1944. Nous ne retenons, pour cette nouvelle estimation, que les individus de la génération 1944 qui disposeraient du même niveau de SAM, quelle que soit la méthode de calcul (8).

En neutralisant l'effet potentiel de l'augmentation du SAM, la surcote continue d'avoir un impact positif sur les comportements de départ en retraite de la génération 1944 (cf. tableau 5). Les individus nés en 1944, ayant validé au moins 160 trimestres à 60 ans et n'étant pas impactés par la modification du SAM (comparativement à ceux nés en 1938), verraient ainsi leur âge de départ en retraite augmenter de cinq mois sous l'effet de la surcote. Les mêmes individus auraient également une probabilité de poursuivre une activité après 60 ans plus élévée de $25 \%$. L’impact est ainsi plus marqué sur cette sous-population d'assurés, ce qui s'explique certainement par sa composition. Les individus non concernés par le changement de SAM sont principalement des individus se situant au deux extrémités de l'échelle des pensions. Ces derniers sont potentiellement plus sensibles aux incitations financières : les assurés aux pensions faibles afin d'améliorer le niveau de leurs droits ; les individus aux pensions élevées pour se maintenir en emploi (effet revenu et meilleures conditions de travail). Les individus composant ce sous-échantillon présentent donc des caractéristiques très particulières. Les résultats présentés ici sont par conséquent difficilement comparables à ceux obtenus sur l'ensemble des individus éligibles à la surcote et doivent être uniquement interprétés comme les résultats d'un test de robustesse à la neutralisation de la différence induite par la réforme de 1993.

La neutralisation de l'effet de la modification du salaire annuel moyen n'annule donc pas l'augmentation de l'âge de la retraite et celle de la probabilité de poursuivre une activité après 60 ans estimées pour l'ensemble des individus nés en 1944 éligibles à la surcote. Il semble donc, à l'instar de l'hypothèse de Bozio (2010), que la modification du SAM ne produise pas de variations importantes des incitations entre des générations relativement proches, a fortiori pour la génération 1944 qui se voit appliquer les mécanismes compensatoires introduits par la réforme de 2003.

\section{Ces effets de la surcote sont-ils spécifiques aux individus nés en 1944, premiers concernés par le dispositif?}

Afin de vérifier que les résultats positifs de la surcote sur les comportements de départ en retraite obtenus en analysant les décisions de départ des assurés nés en 1944 ne sont pas spécifiques à cette génération, nous avons estimé l'impact de la création de la surcote sur d'autres générations. Plus précisément, nous avons procédé à l'application de la même méthode d'évaluation pour les générations 1944, 1945, 1946

8. Les résultats de cette simulation montrent que pour $62 \%$ des individus nés en 1944 et présents dans l'échantillon au 20 ème des assurés de la Cnav, le niveau de leur pension seraient identique avec les deux méthodes de calcul.

\section{Tableau 5 \\ Effet de la surcote sur l'âge de liquidation des droits à la retraite et la probabilité de prolonger son activité après 60 ans au-delà de la durée requise, hors effet du salaire annuel} moyen

\begin{tabular}{|c|c|c|c|}
\hline & $\begin{array}{c}\text { Génération } \\
1944\end{array}$ & $\begin{array}{c}\text { Génération } \\
1938 \\
\text { (contrefactuel) }\end{array}$ & Différence \\
\hline \multicolumn{4}{|c|}{ Âge de liquidation des droits à la retraite } \\
\hline \multirow[t]{2}{*}{$\begin{array}{l}\text { Avant } \\
\text { appariement }\end{array}$} & 61,229 & 60,334 & \\
\hline & & & $(0,029)$ \\
\hline \multirow[t]{2}{*}{$\begin{array}{l}\text { Après } \\
\text { appariement }\end{array}$} & 61,230 & 60,771 & $0,459^{\cdots \cdots}$ \\
\hline & & & $(0,164)$ \\
\hline \multicolumn{4}{|c|}{ Probabilité de prolonger son activité après 60 ans } \\
\hline $\begin{array}{l}\text { Avant } \\
\text { appariement }\end{array}$ & 0,641 & 0,374 & $\begin{array}{l}0,267 \cdots \\
(0,011)\end{array}$ \\
\hline \multirow[t]{2}{*}{$\begin{array}{l}\text { Après } \\
\text { appariement }\end{array}$} & 0,639 & 0,512 & $0,127^{*}$ \\
\hline & & & $(0,062)$ \\
\hline Effectifs & 2722 & 6577 & \\
\hline
\end{tabular}

Lecture : la différence est significative à : ${ }^{* *} 1 \%,{ }^{* *} 5 \%,{ }^{*} 10 \%$, écart-types entre parenthèses.

Champ : assurés nés en 1938 ayant validé au moins 160 trimestres à 60 ans et nés en 1944 ayant validé au moins 160 trimestres à 60 ans et n'étant pas impactés par l'augmentation du nombre de salaires entrant dans le calcul du salaire annuel moyen.

Source : échantillon au $20^{\mathrm{ème}}$ des assurés de la Cnav. 
et 1947. La différence avec l'estimation sur la seule génération 1944 repose sur le fait que les individus composant les générations suivantes sont nombreux à ne pas encore avoir pris leur retraite. On ne peut donc pas observer l'ensemble de leurs décisions de départ. On peut toutefois déjà vérifier s'ils sont plus nombreux à décaler leur départ à la retraite après 60 ans. Une difficulté supplémentaire réside dans le fait que les générations 1945 et suivantes disposant d'une durée validée importante sont non seulement concernées par la création de la surcote mais également par la mise en place du dispositif de retraite anticipée qui permet aux assurés, sous certaines conditions, de liquider leur retraite avant l'âge de 60 ans. Pour contourner cette difficulté, nous proposons d'estimer l'effet causal de la surcote en ne retenant que les individus éligibles à la surcote mais ne pouvant prétendre à la retraite anticipée (9). Cela conduit de nouveau à restreindre l'estimation à une population particulière. De ce fait, les résultats présentés ici ne peuvent être directement comparés à ceux obtenus pour les seules générations 1938 et 1944. Les générations contrefactuelles sont les générations 1938, 1939, 1940 et 1941. Même si certaines d'entre elles sont concernées par l'introduction de la surcote (la génération 1941 à son $63^{\text {ème }}$ anniversaire et la génération 1940 à son 64 ${ }^{\text {ème }}$ anniversaire), leur décision de repousser la liquidation de leurs droits à la retraite après 60 ans est intervenue avant la mise en place de la surcote (respectivement en 2000 et 2001 pour les générations 1940 et 1941). De ce fait, leur probabilité de partir en retraite après 60 ans ne peut pas être imputable à la mise en place de la surcote.

Une fois ces précautions prises et en prenant en compte des caractéristiques observables par le score de propension (sur la même base que les générations 1938 et 1944), on peut suppo-

\section{Tableau 6}

Effet de la surcote sur la probabilité de liquider sa retraite après le $1^{\text {er }}$ trimestre des 60 ans et au-delà de la durée requise pour le taux plein

\begin{tabular}{|l|c|c|c|}
\hline & $\begin{array}{c}\text { Générations } \\
\mathbf{1 9 4 4 - 4 7}\end{array}$ & $\begin{array}{c}\text { Générations } \\
\mathbf{1 9 3 8 - 4 1} \\
\text { (contrefactuel) }\end{array}$ & Différence \\
\hline $\begin{array}{l}\text { Avant } \\
\text { appariement }\end{array}$ & 0,473 & 0,443 & $0,030^{\cdots}$ \\
\hline $\begin{array}{l}\text { Après } \\
\text { appariement }\end{array}$ & 0,472 & 0,364 & $0,108^{\cdots}$ \\
\hline Effectifs & 25478 & 16483 & \\
\hline
\end{tabular}

Lecture : la différence est significative à : *** $1 \%$, ${ }^{* *} 5 \%,{ }^{*} 10 \%$. Champ : générations $1938-41$ et 1944-47 ayant validé au moins 160 trimestres à 60 ans et non éligibles à la retraite anticipée. Source : échantillon au $20^{\text {ème }}$ des assurés de la Cnav. ser que la différence de probabilité de partir en retraite après 60 ans observée entre les générations 1938-1941 et les générations 1944-1947 est imputable à la mise en place de la surcote (cf. tableau 6). La probabilité de repousser la liquidation de la pension du régime général après le $1^{\text {er }}$ trimestre du $60^{\text {ème }}$ anniversaire augmente fortement sous l'effet de l'introduction de la surcote. Les générations 1944, 1945, 1946 et 1947 ont ainsi une probabilité de liquider leur retraite après 60 ans de l'ordre de $47 \%$. Cette probabilité aurait été de $36 \%$ en l'absence de mise en place de la surcote. Contrairement à l'analyse sur la seule génération 1944, l'impact mesuré par appariement sur le score de propension est beaucoup plus important que celui obtenu par différence simple, avec une augmentation de près de $30 \%$ contre $7 \%$ sans prise en compte des caractéristiques observables.

Là encore, comme pour la génération 1944, on trouve un impact plus marqué pour les monopensionnés (26\%), les assurés n'ayant pas connu de chômage entre 55 et 60 ans $(45 \%)$ ou encore ceux ayant validé 41 annuités à 60 ans (70 \%) (cf. graphique V). En revanche, la différence entre hommes et femmes est moins marquée. L'effet estimé de la surcote sur la probabilité de liquider leur retraite après 60 ans est de l'ordre de respectivement $34 \%$ et $29 \%$.

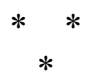

Le dispositif de la surcote, créé en 2004 dans le but d'inciter les futurs retraités à poursuivre leur activité au-delà de la durée requise pour obtenir une retraite au taux plein et à reculer leur âge de départ en retraite, semble donc avoir infléchi les comportements de retraite dans le sens attendu. L'ampleur de cette inflexion reste toutefois limitée, comme le laissait présager la première étude portant sur ce dispositif(Albert et al., 2008), nettement en-deçà de l'effet à attendre d'un allongement de la durée d'assurance requise (Bozio, 2010 et Aubert, 2010). Les résultats concernent par ailleurs une catégorie particulière d'assurés. L'évaluation a en effet été menée sur des individus disposant d'une carrière longue, éligibles à la surcote dès leur $60^{\text {ème }}$ anniversaire. Pour cette raison, l'estimation proposée ici doit être considérée pour ce qu'elle est : une estimation à un niveau local, sur une population spécifique. Elle n'est valable que pour les assurés qui se trou-

9. Les critères d'éligibilité à la retraite anticipée au titre des carrières longues sont plus restrictifs que ceux de la surcote. La durée requise est en effet plus importante et les trimestres pris en compte sont décomposés entre ceux cotisés et ceux validés, les derniers n'étant retenus que jusqu'à une certaine limite. 
vent dans la même situation de départ que ceux que nous avons soumis à l'évaluation. Pour la génération 1944, le critère de sélection retenu est la validation, hors majoration de durée d'assurance, d'au moins 160 trimestres l'année du $60^{\mathrm{ème}}$ anniversaire. Ce critère conduit à ne retenir qu'un tiers des assurés de cette génération. Autrement dit, seulement un tiers des assurés du régime général nés en 1944 se trouvaient en situation de bénéficier de la surcote l'année de leurs 60 ans selon le critère retenu, ce tiers se distinguant nettement des autres assurés par de nombreuses caractéristiques. La proportion d'assurés éligibles à la surcote est par ailleurs amenée à se réduire, sous l'effet de l'augmentation de l'âge d'ouverture des droits à la retraite, de l'allongement de la durée d'assurance requise pour bénéficier d'une retraite au taux plein, du recul de l'âge de fin d'études ou encore de l'accroissement de la probabilité de survenue d'aléas de carrière.

Cette étude d'impact de la surcote ne constitue pas une évaluation complète du dispositif. Il serait nécessaire pour cela de répondre à une série de questions qui restent en suspens à l'is- sue de ce travail. D'abord, il faudrait disposer de davantage de recul pour mesurer réellement l'efficacité du dispositif. Il serait ainsi souhaitable d'observer l'ensemble des comportements de départ en retraite de plusieurs cohortes d'assurés. Cela permettrait de vérifier qu'il ne s'agit pas là que d'effets de court terme et que la mise en place de la surcote a bien conduit à une inflexion importante et durable des comportements de départ en retraite. Il faudrait également s'interroger sur l'articulation de la surcote avec une législation qui évolue, notamment en termes d'âge légal de départ en retraite et de création de dispositifs potentiellement concurrents (comme la libéralisation du cumul emploi-retraite par exemple). Enfin, cette étude ne traite pas de la question pourtant cruciale du choix du barème de la surcote. On peut s'interroger sur le seuil de majoration qui pourrait contribuer plus fortement à modifier les comportements de départ en retraite (Afsa, 2008) sans menacer les comptes des régimes. La question de l'augmentation du barème de la surcote à un niveau plus élevé, comme aux États-Unis par exemple, reste ainsi centrale pour les futurs retraités mais également pour l'équilibre financier des régimes de retraite.

\section{Graphique V \\ Probabilité de liquider sa retraite après le $1^{\mathrm{er}}$ trimestre des 60 ans et au-delà de la durée requise pour le taux plein après estimation par appariement}

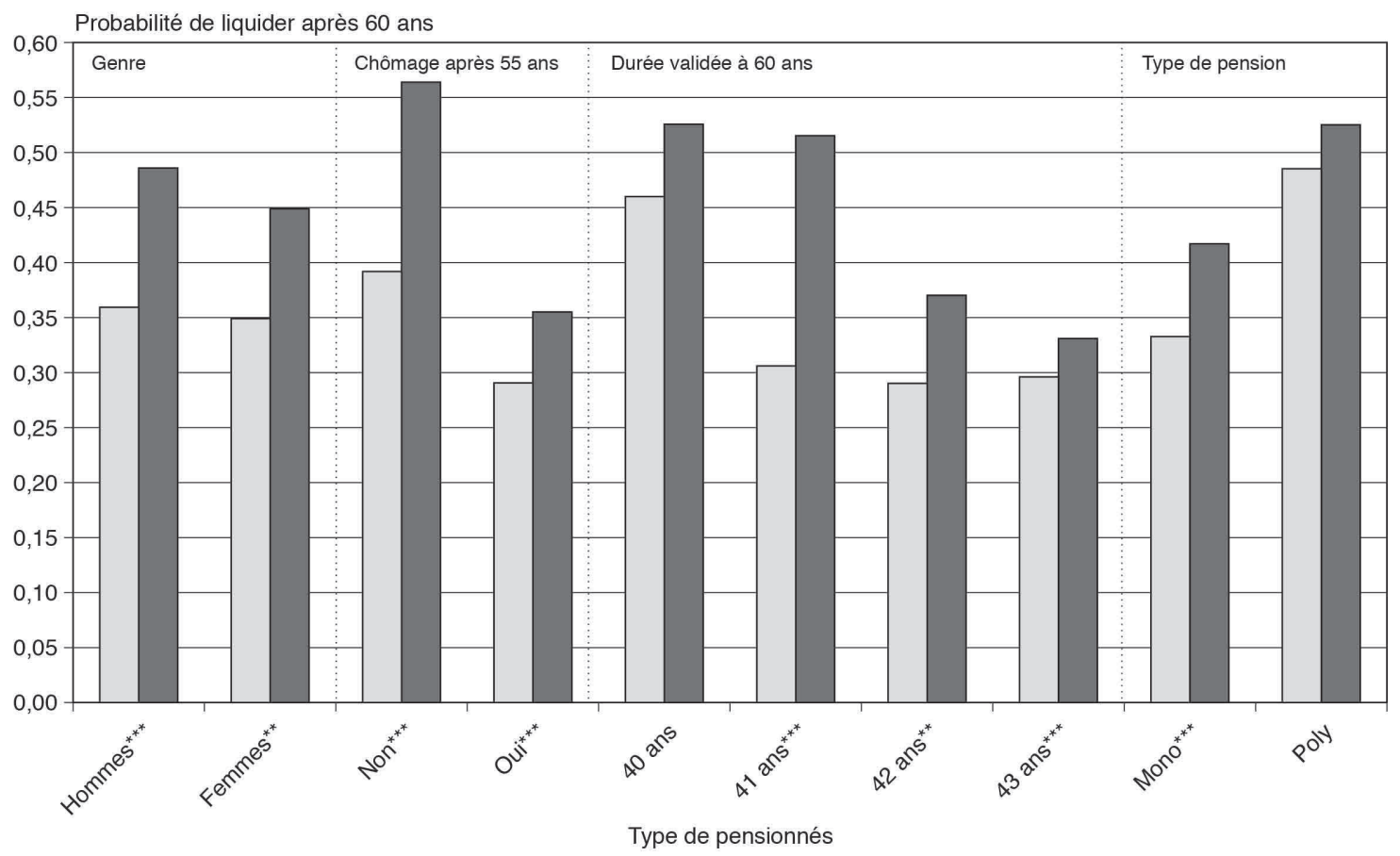

Lecture : la probabilité de prolonger son activité au-delà de 60 ans des hommes des générations 1944 à 1947 est de 48,5\%. Elle aurait été de $36,1 \%$ si la surcote n'avait pas été mise en place. L'estimateur est significatif à : *** $1 \%$, ** $5 \%$, ${ }^{*} 10 \%$.

Champ : générations 1938 à 1947 ayant validé au moins 160 trimestres à 60 ans et non éligibles à la retraite anticipée, respectivement 16483 et 25478 individus.

Source : échantillon au $20^{\text {ème }}$ des assurés de la Cnav. 


\section{BIBLIOGRAPHIE}

Afsa C. (2008), « Estimer la valeur monétaire de la qualité d'un emploi. L'exemple des salariés en fin de carrière ", Revue Économique, vol. 58, $\mathrm{n}^{\circ} 3$, pp. 595-607.

Albert C., Grave N. et Oliveau J.-B. (2008), « Surcote : les raisons d'un échec relatif», Retraite et Société, n 54, pp. 34-63.

Aubert P. (2009), «Allongement de la durée requise pour le taux plein et âge de départ en retraite des salariés du secteur privé : une évaluation de l'impact de la réforme des retraites de $1993 »$, Document de travail du CREST, n 2009-21.

Baraton M., Beffy $M$ et Fougère D. (2010), «Une évaluation de l'effet de la réforme des retraites de 2003 sur les départs en retraite : Le cas des enseignants du second degré public », Document de travail de l'INSEE, ${ }^{\circ}$ G2010-12.

Baker M. et Benjamin D. (1999), « How do retirement tests affect the labour supply of older men ? », Journal of Public Economics, vol. 71, pp. 27-51.

Benallah S. (2009), "Simulation de la prise en compte de l'ensemble des salaires pour le calcul du salaire annuel moyen ", Étude CNAV $n^{\circ} 2009$ 31-DSP, Document de travail du COR $n^{\circ} 9$, séance du 13 mai 2009 : Solidarité et contributivité dans les systèmes de retraite français et étrangers.

Benallah S. et Mette C. (2009), « Âge moyen de départ en retraite : tendances récentes et évolutions attendues », Retraite et Société, $\mathrm{n}^{\circ}$ 58, pp. 166-183.

Benallah S. (2010), « La surcote modifie-t-elle les comportements de départ en retraite ? ", Les Cahiers de la $C N A V, \mathrm{n}^{\circ} 2$.

Bozio A. (2006), « Les réformes des retraites de 1993 et 2003 vont-elles conduire à un allongement des carrières professionnelles ? ", CEPREMAP Docweb $n^{\circ} 0605$.

Bozio A. (2009), «Évaluation de la réforme des retraites de 1993 : nouvelles estimations à partir des données de l'EIR et de l'EIC », Document de Travail de la DREES, Séries Études et Recherches, $\mathrm{n}^{\circ} 91$.

Bozio A. (2010), « Mesurer l'impact de l'augmentation de la durée d'assurance : le cas de la réforme des retraites de $1993 »$, Document de travail du COR $n^{\circ} 11$, séance du 9 juillet 2010 : « Effets des réformes récentes sur les comportements de départ à la retraite ».

Bommier A., Magnac T. et Roger M. (2001), «Départs en retraite : évolutions récentes et modèles économiques », Revue Française d'Économie, vol. $16, \mathrm{n}^{\circ} 1$, pp. $79-124$.

Blöndal S. et Scarpetta S. (1997), « Early retirement in OECD countries : The role of Social Security systems ", OECD Economic Studies, $\mathrm{n}^{\circ} 29$.

Caliendo M. et Kopeinig S. (2005), « Some practical guidance for the implementation of propensity score matching », IZA Discussion Paper Series, $\mathrm{n}^{\circ} 1588$.

Disney R. et Smith S. (2002), «The labour supply effect of the abolition of the earnings rule for older workers in the United Kingdom », The Economic Journal, vol. 112, n 478, pp. C136-C152.

Gruber J. et Orszag P. (2003), « Does the Social Security Earnings test affect labor supply and benefits receipt », National Tax Journal, vol. 56, $\mathrm{n}^{\circ} 4$, pp. 755-773.

Gruber J. et Wise D. (2004), Social Security Programs and Retirement around the World : Micro-simulation, NBER/The University of Chicago Press.

Haider S. et Loughran D. S. (2008), « The Effect of the Social Security Earnings Test on Male Labor Supply : New Evidence from Survey and Administrative Data ", Journal of Human Resources, vol. 43, $\mathrm{n}^{\circ} 1$.

Krueger A. et Pischke J.-S. (1992), « The Effect of Social Security on Labor Supply : A Cohort Analysis of the Notch Generation ", Journal of Labor Economics, vol. 10, $\mathrm{n}^{\circ}$ 4, pp. 412-437.

Leuven E. et Sianesi B. (2003), « PSMATCH2 : Stata module to perform full Mahalanobis and propensity score matching, common support graphing, and covariate imbalance testing ", Software component $n^{\circ}$ S432001, Boston College Department of Economics.

Lumsdaine, R. et Mitchell, O. (1999), « New Developments in the Economic Analysis of 
Retirement », dans O. Ashenfelter et D. Card, eds, Handbook of Labor Economics, vol. 3C, pp. 32613307.

Magnac T., Rapoport B. et Roger M. (2006), « Fins de carrière et départs à la retraite : l'apport des modèles de durée », Dossier Solidarité Santé $\mathrm{n}^{\circ} 3$, pp. 101-117.

Mastrobuoni G. (2009), « Labor supply effects of the recent Social Security benefit cuts : empirical estimates using cohort discontinuities », Journal of Public Economics, vol. 93, pp. 12241233.

Pingle J. F. (2006), « Social Security's delayed retirement credit and the labor supply of older men », FEDS Working paper, n 2006-37.

Rosenbaum P. et Rubin D. (1983), « The central role of the propensity score in observational stu- dies for causal effects », Biometrika, vol. 70, $\mathrm{n}^{\circ} 1$, pp. 41-55.

Rubin D. (1974), « Estimating causal effects of treatments in randomized and nonrandomized studies ", Journal of Educational Psychology, vol. $66, \mathrm{n}^{\circ} 5$, pp. 688-701.

Song J. G. (2003/2004), « Evaluating the initial impact of the elimination of the retirement earnings test », Social Security Bulletin, vol. 65, $\mathrm{n}^{\circ} 1$, pp. $1-15$.

Song J. et Manchester J. (2007a), « New evidence on earnings and benefit claims following changes in the retirement earnings test in 2000 ", Journal of Public Economics, vol. 91, pp. 669700.

Song J. et Manchester J. (2007b), « Have people delayed claiming retirement benefits ? Responses to changes in Social Security Rules », Social Security Bulletin, vol. 67, $\mathrm{n}^{\circ} 2$. 
Tableau A

Caractéristiques entrant dans le calcul du score de propension

\begin{tabular}{|c|c|c|}
\hline Caractéristiques & Description & Modalités \\
\hline \multicolumn{3}{|c|}{ Sociodémographiques } \\
\hline SEXE & Sexe & Hommes ; Femmes \\
\hline PAYS_NAIS & Pays de naissance & France ; Étranger \\
\hline \multicolumn{3}{|l|}{ Revenus } \\
\hline SAM_10 & $\begin{array}{l}\text { Moyenne des } 10 \text { meilleures années de salaire à l'âge } \\
\text { de } 60 \text { ans }\end{array}$ & $\begin{array}{l}\text { Classes par quartiles de salaires. Les quartiles sont } \\
\text { calculés séparément pour les hommes et les femmes }\end{array}$ \\
\hline \multicolumn{3}{|l|}{ Carrière } \\
\hline RG & $\begin{array}{l}\text { Part du régime général dans les trimestres cotisés à } \\
\text { l'âge de } 55 \text { ans }\end{array}$ & $\begin{array}{l}30 \% \text { ou moins ; Entre } 30 \% \text { et } 50 \% \text { de la carrière ; } \\
\text { Entre } 50 \% \text { et } 80 \% \text {; Entre } 80 \% \text { et } 100 \% ; 100 \% \text { de } \\
\text { la carrière }\end{array}$ \\
\hline CHO_55 & $\begin{array}{l}\text { Part du chômage dans l'ensemble de la carrière à } \\
\text { l'âge de } 55 \text { ans }\end{array}$ & $\begin{array}{l}\text { Aucune période de chômage ; entre } 0 \text { et } 5 \% \text { de la } \\
\text { carrière; entre } 5 \text { et } 10 \% \text { de la carrière ; Plus de } 10 \% \\
\text { de la carrière }\end{array}$ \\
\hline \multicolumn{3}{|c|}{ Situation entre 55 et 60 ans } \\
\hline MAL_55_60 & Nombre de trimestres de maladie entre 55 et 60 ans & $\begin{array}{l}\text { Aucune période de maladie ; Au moins un trimestre } \\
\text { de maladie }\end{array}$ \\
\hline CHO_55_60 & Nombre de trimestres de chômage entre 55 et 60 ans & $\begin{array}{l}\text { Aucune période de chômage ; Entre } 1 \text { et } 8 \text { trimestres ; } \\
\text { Plus de } 8 \text { trimestres }\end{array}$ \\
\hline \multicolumn{3}{|l|}{ Santé } \\
\hline MAL_55 & $\begin{array}{l}\text { Part des périodes de maladie dans l'ensemble de la } \\
\text { carrière jusqu'à l'âge de } 55 \text { ans }\end{array}$ & $\begin{array}{l}\text { Aucune période de maladie ; Entre } 0 \text { et } 2 \% \text { de la } \\
\text { carrière ; Plus de } 2 \% \text { de la carrière }\end{array}$ \\
\hline \multicolumn{3}{|c|}{ Longueur de la carrière } \\
\hline VALI_60 & Nombre de trimestres validés à 60 ans & $\begin{array}{l}\text { Entre } 160 \text { et } 163 \text { trimestres; entre } 164 \text { et } 167 \text { trimestres ; } \\
\text { entre } 168 \text { et } 172 \text { trimestres; Plus de } 172 \text { trimestres }\end{array}$ \\
\hline
\end{tabular}

Source : auteure.

Tableau B

Estimations du score de propension $e(X)$, modèles Probit

\begin{tabular}{|c|c|c|c|}
\hline \multicolumn{2}{|c|}{ Caractéristiques } & Génération 1944 & Générations 1941 à 1947 \\
\hline \multicolumn{4}{|c|}{ Sociodémographiques } \\
\hline SEXE & $\begin{array}{l}\text { Homme } \\
\text { Femme }\end{array}$ & $\begin{array}{c}-0,089^{* * *} \\
(0,025) \\
\text { Réf. }\end{array}$ & $\begin{array}{c}-0,207^{* *} \\
(0,014) \\
\text { Réf. }\end{array}$ \\
\hline PAYS_NAIS & $\begin{array}{l}\text { France } \\
\text { Étranger }\end{array}$ & $\begin{array}{c}-0,341^{*+*} \\
(0,057) \\
\text { Réf. }\end{array}$ & $\begin{array}{c}-0,025^{+* *} \\
(0,028) \\
\text { Réf. }\end{array}$ \\
\hline \multicolumn{4}{|l|}{ Revenus } \\
\hline SAM_10 & $\begin{array}{l}1^{\text {er }} \text { quartile } \\
\text { 2è quartile } \\
\text { 3è quartile } \\
\text { 4è quartile }\end{array}$ & $\begin{array}{c}-0,778^{* * *} \\
(0,057) \\
-0,876^{* * *} \\
(0,034) \\
-0,848^{* * *} \\
(0,028) \\
\text { Réf. }\end{array}$ & $\begin{array}{c}-0,866^{* * *} \\
(0,036) \\
-0,889^{* * *} \\
(0,220) \\
-0,858^{* * *} \\
(0,018) \\
\text { Réf. }\end{array}$ \\
\hline
\end{tabular}




\begin{tabular}{|c|c|c|c|}
\hline \multicolumn{2}{|c|}{ Caractéristiques } & Génération 1944 & Générations 1941 à 1947 \\
\hline \multicolumn{4}{|l|}{ Carrière } \\
\hline $\mathrm{RG}$ & $\begin{array}{l}\text { Au plus } 30 \% \\
\text { Entre } 30 \% \text { et } 50 \% \\
\text { Entre } 50 \% \text { et } 80 \% \\
\text { Entre } 80 \% \text { et } 100 \% \\
100 \%\end{array}$ & $\begin{array}{c}\text { Réf. } \\
-0,1966^{\cdots *} \\
(0,055) \\
-0,525^{\cdots} \\
(0,060) \\
-0,6644^{\cdots *} \\
(0,061) \\
-0,856 \cdots \\
(0,058)\end{array}$ & $\begin{array}{c}\text { Réf. } \\
-0,171^{\cdots *} \\
(0,036) \\
-0,052^{\cdots *} \\
(0,039) \\
-0,069 \cdots \\
(0,039) \\
-0,911^{\cdots} \\
(0,037)\end{array}$ \\
\hline CHO_55 & $\begin{array}{l}\text { Aucune période de chômage } \\
\text { Entre } 0 \text { et } 5 \% \\
\text { Entre } 5 \% \text { et } 10 \% \\
\text { Plus de } 10 \%\end{array}$ & $\begin{array}{c}\text { Réf. } \\
0,432 \\
(0,102) \\
0,586 \\
(0,126) \\
0,931^{\cdots *} \\
(0,114) \\
\end{array}$ & $\begin{array}{c}\text { Réf. } \\
0,273^{\cdots+} \\
(0,058) \\
0,507{ }^{\cdots *} \\
(0,068) \\
0,851^{\cdots} \\
(0,069) \\
\end{array}$ \\
\hline PAYS_NAIS x CHO_55 & $\begin{array}{l}\text { France } x \text { entre } 0 \text { et } 5 \% \\
\text { France } x \text { entre } 5 \% \text { et } 10 \% \\
\text { France } x \text { plus de } 10 \%\end{array}$ & $\begin{array}{c}-0,065 \\
(0,107) \\
-0,051 \\
(0,132) \\
0,052 \\
(0,120) \\
\end{array}$ & $\begin{array}{r}0,066 \\
(0,062) \\
-0,010 \\
(0,071) \\
0,113^{*} \\
(0,061)\end{array}$ \\
\hline \multicolumn{4}{|c|}{ Situation entre 55 et 60 ans } \\
\hline MAL_55_60 & $\begin{array}{l}\text { Aucun trimestre de maladie } \\
\text { Au moins un trimestre de } \\
\text { maladie }\end{array}$ & $\begin{array}{c}\text { Réf. } \\
0,158 \cdots \\
(0,033)\end{array}$ & $\begin{array}{c}\text { Réf. } \\
0,100 * \\
(0,022)\end{array}$ \\
\hline CHO_55_60 & $\begin{array}{l}\text { Aucun trimestre de chômage } \\
\text { Entre } 1 \text { et } 8 \text { trimestres } \\
\text { Plus de } 8 \text { trimestres }\end{array}$ & $\begin{array}{c}\text { Réf. } \\
-0,4544^{-*} \\
(0,033) \\
-0,345^{\cdots *} \\
(0,027)\end{array}$ & $\begin{array}{c}\text { Réf. } \\
-0,199 * \cdots \\
(0,04) \\
-0,228 * \cdots \\
(0,019)\end{array}$ \\
\hline \multicolumn{4}{|l|}{ Santé } \\
\hline \multirow[t]{4}{*}{ MAL_55 } & Aucune période de maladie & Réf. & Réf. \\
\hline & Entre 0 et $2 \%$ & $0,202^{\cdots *}$ & $0,172^{\cdots *}$ \\
\hline & & $\begin{array}{l}(0,028) \\
\end{array}$ & $\begin{array}{l}(0,017) \\
\end{array}$ \\
\hline & . & $(0,036)$ & $(0,021)$ \\
\hline \multicolumn{4}{|c|}{ Nombre de trimestres validés à 60 ans } \\
\hline VALI_60 & $\begin{array}{l}\text { Entre } 160 \text { et } 163 \text { trimestres } \\
\text { Entre } 164 \text { et } 167 \text { trimestres } \\
\text { Entre } 168 \text { et } 172 \text { trimestres } \\
\text { Plus de } 172 \text { trimestres }\end{array}$ & $\begin{array}{c}\text { Réf. } \\
-0,023 \\
(0,033) \\
0,109 * * \\
(0,031) \\
0,142^{* * *} \\
(0,032)\end{array}$ & $\begin{array}{c}\text { Réf. } \\
-0,021 \\
(0,015) \\
0,000 \\
(0,022) \\
0,196 * \cdots \\
(0,026)\end{array}$ \\
\hline \multicolumn{2}{|l|}{ Constante } & $\begin{array}{c}1,637 \\
(0,073)\end{array}$ & $\begin{array}{c}1,673^{\cdots \prime} \\
(0,045)\end{array}$ \\
\hline Observations & & 16397 & 41961 \\
\hline
\end{tabular}

Lecture : le coefficient est significatif à : *** $1 \%,{ }^{* *} 5 \%$, $10 \%$, écart-type entre parenthèses.

Champ : générations 1938 et 1944 ayant validé au moins 160 trimestres à 60 ans et générations 1938 à 1947 ayant validé au moins 160 trimestres à 60 ans et non éligibles à la retraite anticipée.

Source : échantillon au $20^{\text {ème }}$ des assurés de la Cnav. 